\title{
Professional improvising musicians couple more closely in timing and tonality when simultaneously rather than successively recording
}

\author{
Matthew Setzler ${ }^{1,2}$ and Robert L. Goldstone ${ }^{1}$ \\ ${ }^{1}$ Department of Psychological and Brain Sciences, Program in Cognitive Science, Indiana University, Bloomington, IN, USA
}

This manuscript was compiled on February 20, 2020

\begin{abstract}
Joint action (JA) is ubiquitous in our cognitive lives. From basketball teams to teams of surgeons, humans often coordinate with one another to achieve some common goal. Despite this ubiquity, the individual mechanisms and group-level dynamics of complex, sophisticated JA are poorly understood. We examine coordination in a paragon domain for creative joint expression: improvising jazz musicians. Coordination in jazz music is improvised and subserves an aesthetic goal: the generation of a collective musical expression comprising coherent, highly nuanced musical structure (e.g. rhythm, harmony). In this study, dyads of professional jazz pianists improvised in a "coupled", mutually adaptive condition, and an "overdubbed" condition which precluded mutual adaptation, as occurs in common studio recording practices. Using a model of musical tonality, we quantify the flow of rhythmic and harmonic information between musicians as a function of interaction condition, and show that mutually responsive dyads produce more consonant harmonies, an ability which increases throughout the course of improvised performance. These musical signatures of coordination were paralleled in the subjective experience of improvisers, who preferred coupled trials despite being blind to condition. We present these results and discuss their implications for music technology and JA research more generally.
\end{abstract}

joint action | improvisation | time series modeling | music

Joint action (JA) is ubiquitous in our cognitive lives. From basketball teams to teams of surgeons, humans often coordinate with others towards a common goal $(1,2)$. In such settings, group behavior is less dependent on isolated individual efforts and more on a group's ability to successfully coordinate $(3,4)$. JA has important implications for our understanding of interpersonal interaction, optimizing team performance, and engineering human-like artificial intelligence systems (5-8). This study examines JA in collaboratively improvising jazz musicians. Like many forms of JA (e.g. conversation), coordination in jazz music is improvised and subserves an aesthetic goal: the generation of a collective musical expression. Moreover, professional jazz musicians dedicate the better part of their lives to developing the ability to adapt and coordinate in real time improvised performance. Professional jazz ensembles thus offer a rarefied, remarkably sophisticated paragon domain in which we can study the basic properties and limits of our capacity to coordinate with one another.

Musicians respond and adapt to one another when they play together. These interactions are mediated by organizational structures which can vary depending on genre, performance/recording context and personnel. For example, orchestras are hierarchically organized with prescribed leaderfollower roles fixed throughout a performance, whereas free improvising jazz ensembles are typically more characterized by feedback loops of mutual influence $(9,10)$. Ensemble performance research has shown that these underlying patterns of coordination are reflected in synchrony and entrainment of ensemble members (11-13). For example, small temporal asynchronies in co-performer note onsets have been shown to reflect leader-follower roles and the degree to which musicians mutually adapt to one another (14-16), and postural sway couplings reflect leader-follower relations in ensembles (17).

Improvised music is of particular interest, because the influence of coordination extends beyond sensorimotor coupling and into the music's formal architecture, which is freely evolving over time in its rhythm, melody, harmony, and texture. These components of musical structure are fixed in composed music, but collectively generated in real time improvised performance. Previous studies have shed light on the individual-level neurocognitive mechanisms supporting musical improvisation (18-21), but here we are interested in how group-level dynamics emerge out of underlying musical interactions and coordination patterns. How do coordination patterns constrain music produced by improvising ensembles? Developing musically relevant measures of improvised coordination can inform the development of artificial interactive music systems (22) and benefit music pedagogy by automating assessment of ensemble performance. More broadly, it offers an important extension to human interaction research, which has focused largely on various aspects of sensorimotor coordination such as entrainment, mimicry and coupling of body dynamics (23-26), but less on the mechanisms and dynamics of coordination as it occurs in abstract spaces (e.g. aligning mental representations in conversation or generating new ideas in group brainstorming sessions) $(27,28)$.

Measuring aspects of music that are structurally deep, nuanced and psychologically resonant is computationally tractable thanks to research over centuries on music theory and decades on Music Information Retrieval. In the present study we focus on two fundamental musical features: onset density and tonal consonance. Onset density indexes overall rhythmic activity level, and has been shown to correlate with listener perception of musical tension (29). Tonal consonance refers to how different combinations of notes sound on a continuum from dissonant/unstable to consonant/stable (30). Tonal music is essentially a dynamic interplay between these extremes. Remarkably, freely improvising jazz musicians spontaneously generate coherent tonal structure without any prior harmonic

Authors contributed equally to designing the experiment. Matthew Setzler collected data, performed all analyses and wrote the manuscript, with guidance and input from Robert Goldstone.

The authors declare no conflicts of interest.

${ }^{2}$ To whom correspondence should be addressed. E-mail: msetzleriu.edu 
form. We adapt a previously established tonal model - the Tonal Spiral Array (31-33) - to operationalize a measure of tonal consonance. Time series of these features are extracted from individual and collective musical streams from collaboratively improvising jazz musicians playing in experimentally manipulated conditions of interaction.

This present work builds on previous empirical studies which have shown that improvised musical coordination is shaped by musical context (e.g. playing with a drone versus a swing backing track), and that experimentally manipulated social attitudes (e.g. dominant, caring) are sonically encoded and perceivable in the music produced by co-improvising musicians $(34,35)$. These findings lay an important foundation to the study of joint action in improvised music, but since the analyses did not incorporate tonal music theory, the findings are limited to sensorimotor and rhythmic coordination, and do not extend to more sophisticated musical phenomena such as the emergence of tonal structure.

In the current study we directly manipulate interaction of improvising musicians to examine how different underlying patterns of coordination constrain the exchange and emergence of rhythmic and tonal musical information. Dyads of professional jazz musicians freely improvised in two conditions of interaction: (1) a coupled condition, in which pianists improvised simultaneously and (2) a one-way condition, in which a single pianist improvised along with a recording of another pianist (a 'ghost' partner) taken from a previous coupled performance. Musicians could mutually adapt to one another in coupled trials, but the one-way condition enforced an asymmetric causal influence (i.e. from recording to musician), as in the common recording technique of 'overdubbing'. These conditions allowed us to isolate the effects of mutual coupling by contrasting music produced in two naturalistic musical settings. Onset density and tonal consonance time series were extracted from the individual and merged musical streams (recorded as isolated MIDI $^{*}$ tracks for each musician) produced in each condition. We find that interaction condition systematically altered the coordinated musical behavior of dyads, who were more rhythmically coupled and produced more consonant tonal structure which evolved dynamically throughout improvised pieces in coupled trials. These behavioral effects were paralleled in the subjective experiences of participants, who preferred coupled trials despite being blind to condition. These results are presented and discussed in terms of their implications for music technology and JA research more generally.

\section{Results}

Subjective Ratings. Despite being blind to condition, participants systematically preferred coupled trials over one-way trials. Participants rated coupled trials to produce higher quality music (21 out of 26 participants rated coupled higher; probability of success $=0.81$; exact binomial test $\mathrm{p}<.01$ ). Coupled trials were also rated as being better coordinated ( 23 out of 26 participants rated coupled trials as being better coordinated; probability of success $=0.88$; binomial test $\mathrm{p}<.01$ ), and more readily coordinated ( 24 out of 26 subjects found it easier to coordinate with their partner in coupled trials; probability $.92 ; \mathrm{p}<.01)$. Participants were also asked to rate the degree to which they played a leader versus a follower

* Musical Instrument Digital Interface (MIDI) is a format for representing music on a computer. It symbolically represents the pitch, volume and timing (onset and offset) of musical note sequences. role on a 5-point scale. There was again a significant effect of condition: participants in one-way trials felt they played mostly a supporter role whereas participants in coupled trials mostly rated themselves as neutral (difference between average ratings within participant by condition; paired-t $(25)=3.16$, $\mathrm{p}<.01)$.

Mutual Coupling Promotes Synchrony. How does coupling influence musicians' ability to synchronize their note onsets with one another? Asynchronies between 'near-simultaneous' onsets - those occurring within 100 milliseconds of one another - played by co-performers were measured throughout all trials in each condition, as depicted in Figure 1. Near-zero asynchronies indicate close temporal alignment and thus precise synchronization, while asynchronies of larger magnitude reflect less precise synchronization. Asynchronies in coupled trials are peaked around zero, whereas asynchronies in one-way trials are more widely distributed throughout the $+/-100 \mathrm{~ms}$ range $(\mathrm{KS}$.test $\mathrm{D}=0.024, \mathrm{p}$-value $<.01)$. This indicates that musicians achieved more precise synchronization when they had the ability to mutually adapt to one another, which is consistent with previous findings in the context of composed music $(15,16)$. Previous work has also suggested that humans have an innate predisposition to entrain more effectively to other humans as opposed to machine rhythms(36), which could explain the current results as participants may have sensed a lack of live responsiveness in their partners in one-way trials.

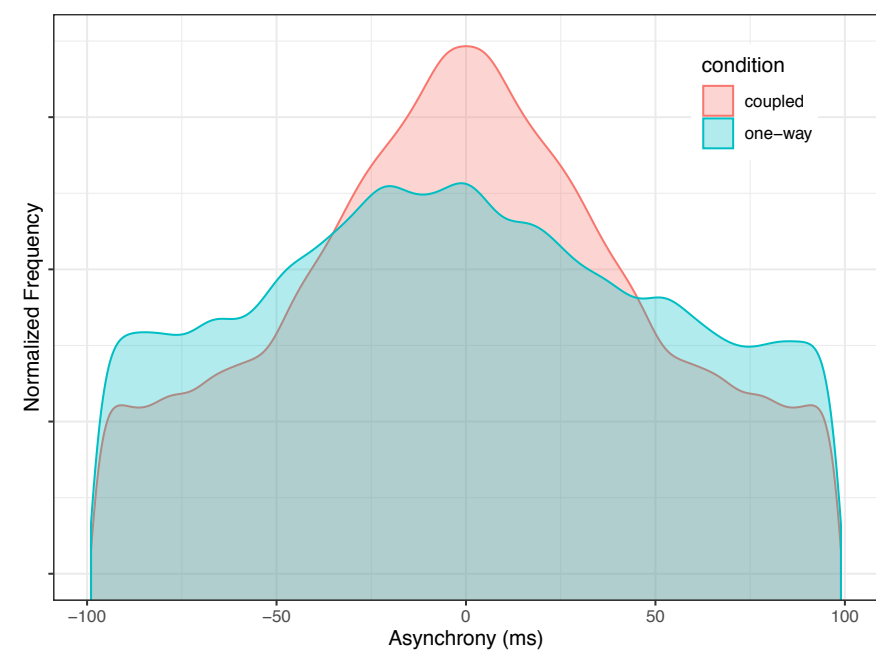

Fig. 1. Mutual coupling facilitates precise synchronization. Distribution of asynchronies between co-performers' near-simultaneous (within $100 \mathrm{~ms}$ ) note onsets throughout all trials in each condition. Asyncs are more tightly clustered around 0 in coupled trials, indicating more precise temporal alignment.

Activity Matching. Lagged cross-correlation of co-performers' onset density was computed to analyze how musicians respond to one another's rhythmic activity level, as depicted in Figure 2. (Onset density time series were computed for each individual musical sequence using a 2 second sliding window, with 0.2 second hop size.) Overall cross-correlation (cc) was positive $(\mathrm{t}(135)=15.1, \mathrm{p}<.01 ;$ mean cc per piece averaged across $+/-20$ second lag range), indicating a general tendency for musicians to match the onset density of their partners. Cross-correlation was higher in coupled versus one-way trials $(\mathrm{t}(99.6)=2.66$; 


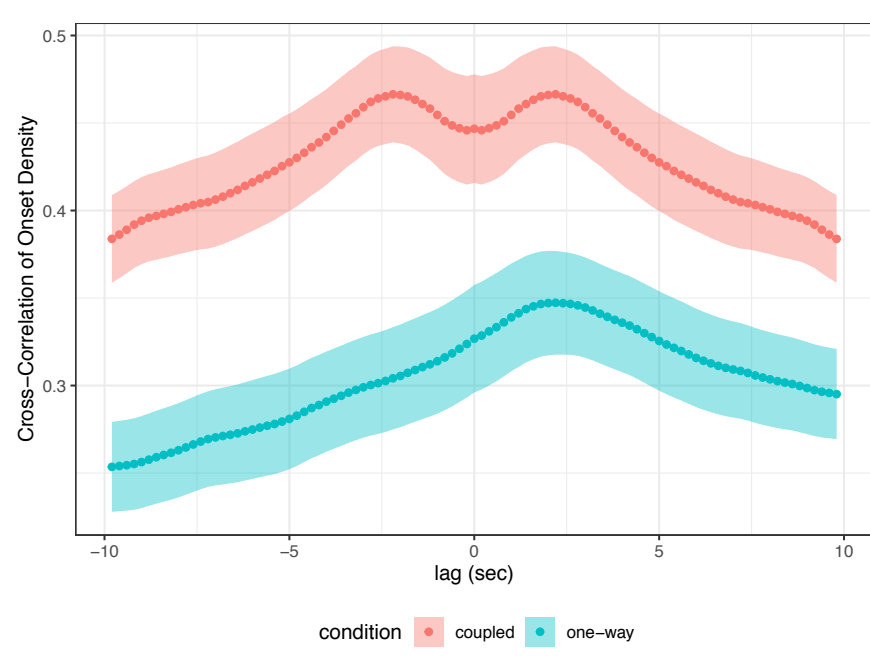

Fig. 2. Musicians match the activity level of their partners. Points represent mean lagged cross-correlation across all trials within each condition, error ribbons denote standard error of the mean. Positive lags in one-way trials represent correlation of ghost recording onset density with the future onset density of live musicians (ghost-tolive) and vice versa for negative lags (live-to-ghost).

$\mathrm{p}<0.01$ ), indicating that this tendency is exaggerated with mutual coupling. Within one-way trials, cc was higher at positive, 'ghost-to-live' lags (onset density ghost recording correlated with future onset density of live musician) versus negative, 'live-to-ghost' lags (paired $\mathrm{t}(85)=5.2 ; \mathrm{p}<0.01$ ). This reflects the underlying asymmetry of one-way trials: live musicians are responsive to notes of the ghost recording but not the other way around. As reported in the SI, this latter result was also observed in a Granger Causality analysis, which is a more stringent test of causality as it separates the causal influence of a source time series (i.e. onset density of a player's partner) from autoregressive predictability within a target time series (i.e. past onset density of the player themselves).

Tonal Coordination. A previously established model of tonal structure (see Methods and SI) was adapted to provide a measure of tonal consonance, quantifying how collections of notes sound on a continuum from unstable/dissonant to stable/consonant $(31,33)$. Time series of Individual Consonance (consonance of individual music streams) and Combined Consonance (consonance of merged music streams from both players in a dyad) were computed with a sliding window. Additionally, Emergent Consonance (EC) was operationalized as Combined Consonance minus average Individual Consonance of both musicians in a dyad. EC is essentially a measure of tonal coordination, as it captures the consonance arising from the interaction of pitches played by the two different musicians. A situation in which each pianist plays consonant notes that clash with one another would result in low EC (e.g. $\{\mathrm{C}, \mathrm{E}, \mathrm{G}\}$ and $\{\mathrm{F} \#, \mathrm{~A} \#, \mathrm{C} \#\}$ are consonant on their own but $\{\mathrm{C}, \mathrm{E}, \mathrm{G}, \mathrm{F} \#, \mathrm{~A} \#, \mathrm{C} \#\}$ is highly dissonant), whereas a situation in which each pianist plays dissonant notes that stabilize one another when sounded together would result in high EC (e.g. $\{\mathrm{C}, \mathrm{B}\}$ and $\{\mathrm{E}, \mathrm{G}\}$ have low average consonance but $\{\mathrm{C}, \mathrm{E}, \mathrm{G}, \mathrm{B}\}$ has high consonance because it is tonicized to a Cmaj7 chord). Negative EC values can be interpreted as emergent dissonance.
Directed Flow of Tonal Information. A novel lagged consonance analysis was used to quantify how musicians responded to and harmonized with one another's notes as a function of interaction condition. Lagged consonance was computed by taking individual note sequences of co-performers, shifting them relative to the other performer by some lag, and computing Combined and Emergent Consonance time series of the merged pitch collections using a sliding window (5 second sliding window, 2 second hop size). This analysis measures the causal influence and directed flow of tonal information, as it quantifies the degree to which individuals harmonize with the preceding notes of their co-performer. For example, Player A might respond and harmonize with Player B's past notes but not the other way around, which would be reflected in high consonance for B-to-A lags but not for A-to-B lags.

Lagged consonance was computed for every trial in each condition with lags in the range of $+/-20$ seconds, spaced by increments of 2 seconds. In one-way trials, positive lags correspond to evaluating past notes of the ghost recording with future notes of the live musician (ghost-to-live) and vice versa for negative lags (live-to-ghost). The beginnings and endings of pieces (first and last 10\%) were discarded to avoid boundary effects. Average Combined and Emergent Consonance were computed at every lag within each trial. The reported results come from using a 5 second sliding window, but these results were robust across a range of window sizes, as reported in the SI.

Figure 3 depicts average Emergent Consonance (EC) across the range of lags by condition. EC is essentially symmetric around 0 for coupled trials, but is significantly higher for ghost-to-live (positive) lags compared to live-to-ghost (negative) lags within one-way trials (paired $\mathrm{t}(85)=2.3, \mathrm{p}<0.05$ mean ghost-to-live compared to mean live-to-ghost EC). This same asymmetry in one-way trials was found with respect to Combined Consonance (paired $\mathrm{t}(85)=2.5, \mathrm{p}<.01$ ). These results reflect the underlying causal entanglements of each condition. Live musicians in one-way trials responded to ghost recordings by harmonizing with their past notes - resulting in high ghost-to-live lagged consonance, but ghost recordings could not respond to notes of the live musicians - resulting in lower, presumably chance-level live-to-ghost lagged consonance. There was no such asymmetry in coupled trials, since both musicians could mutually respond to one another. Interestingly, EC appears to be higher overall in coupled versus one-way trials. This observation is further investigated in the following section.

Emergent Tonal Structure Over Time. How does interaction condition affect dyads' ability to collectively produce tonal structure at the group-level? Time series of lag-0 (simultaneous) Emergent Consonance were computed for every trial in each condition with a 5 second sliding window and 0.2 second hop size. Overall there was more EC in coupled trials versus one-way trials (paired-t $(42)=2.21, \mathrm{p}<0.05$, difference of means -0.011 ; paired t-test comparing mean consonance of each coupled trial versus that of the correspondingly yoked one-way pieces). Mutually adaptive dyads thus exhibited higher tonal coordination compared to overdubbed dyads, as they were better able to consonantly harmonize their notes with one another.

Is there systematic structure to how Emergent Consonance evolves within pieces? If so, is this temporal structure mod- 


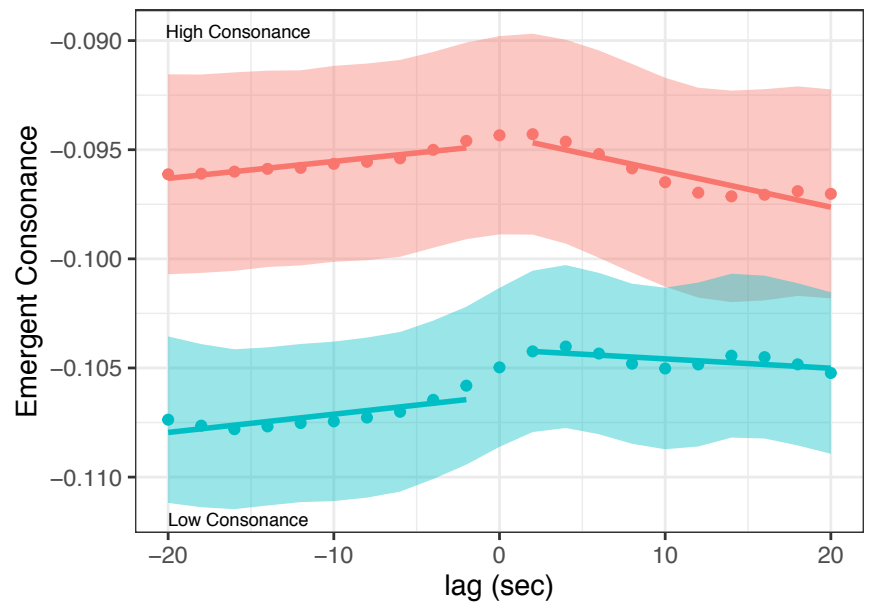

condition coupled one-way

Fig. 3. Lagged consonance analysis reveals musicians harmonize with preceding notes of their partner. Negative lags correspond to notes of the live musician merged with future notes of the ghost recording (live-to-ghost) and vise versa for positive lags (ghost-to-live). Points denote average EC at a given lag across every piece within each condition, error bars denote standard error of the mean. Linear fits of EC by lag are shown for negative and positive lags in each condition.

ulated by interaction condition? We examined EC over normalized time, as the average EC in 50 equispaced time bins for each trial, depicted in Figure 4 (other binning schemes yielded the same results, as described in the SI). Hierarchical Bayesian modeling was used to model the time course of EC as a function of condition. A quadratic model of EC as a function of time was fit for every trial, and trial-level parameters were modeled as being drawn from condition-level distributions. This allowed us to isolate how the time course of consonance was modulated by condition by comparing means of condition-level distributions for each term.

This analysis revealed temporal structure that was common to trials in both conditions, but that was more exaggerated in coupled trials. In both conditions we found a positive linear term (posterior estimate of linear term for coupled trials was $6.4 \mathrm{e}-3$ with $95 \%$ CI [4.6e-3,8.1e-3], with Effective Sample Size $($ ESS $)=7890$, Gelman-Rubin Statistic $=1.00$; estimate of average slope for one-way trials was $4.2 \mathrm{e}-3$ with $95 \%$ CI $[2.9 \mathrm{e}-3,5.5 \mathrm{e}-3], \mathrm{ESS}=8208$, Gelman-Rubin Statistic $=1.00)$ and a negative quadratic term (posterior estimate of quadratic coefficient for coupled trials was -1.1e-4 with [-1.4e-4,-7.6e-5] 95\% CI, ESS $=9170$, Gelma-Rubin Stat $=1.00$; estimate was $-7.7 \mathrm{e}-5$ for one-way trials with $[-1.03 \mathrm{e}-4,-5.1 \mathrm{e}-5] 95 \% \mathrm{CI}, \mathrm{ESS}=8220$ Gelman-Rubin Stat=1.00). The positive linear term indicates a general trend for EC to increase throughout an improvised performance, while the negative quadratic term reflects the extent to which a non-monotonic low-high-low structure for EC is found, which combines additively with the linear relation. Interestingly, the linear term was significantly more positive in coupled trials versus one-way trials (posterior estimate of difference between linear slopes i.e. coupled minus one-way is $2.2 \mathrm{e}-3$ with [1.6e-06,4.3e-03] 95\% CI i.e. non-overlapping with zero, ESS=8171, Gelman-Rubin Stat=1.00). These posterior distributions indicate a general trend for tonal coordination to increase throughout performances, and this increase hap- pens significantly moreso in coupled trials. In addition, both conditions have similar tendencies to first increase and then decrease their Emergent Consonance.

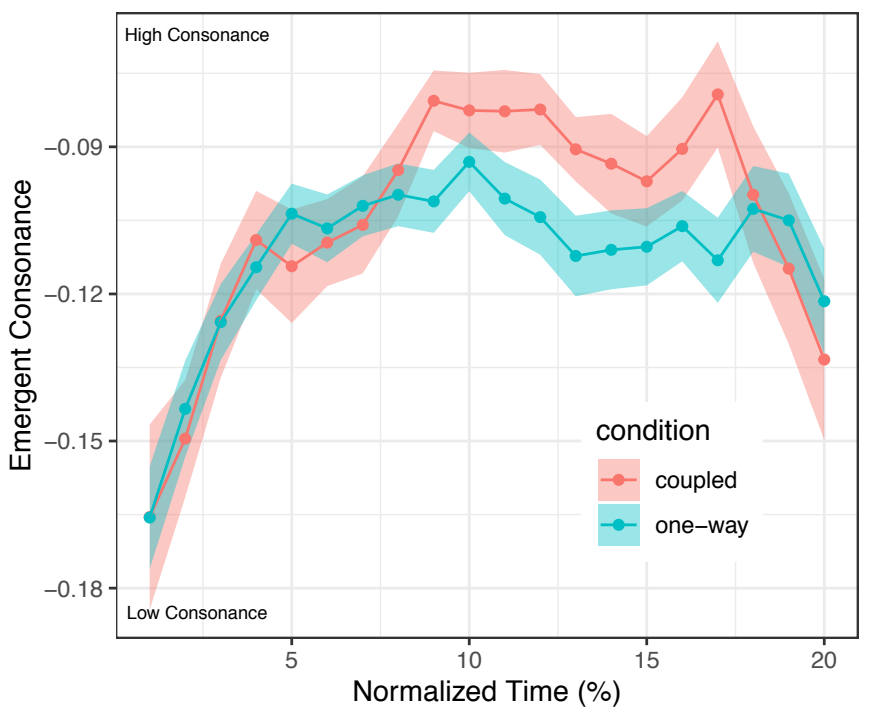

Fig. 4. Systematic structure to Emergent Consonance (EC) over normalized time. Pieces were divided into 20 equispaced time bins. Points denote average EC across all pieces within each condition, error ribbons denote standard error of the mean.

\section{Discussion}

This study examined how the music produced by collectively improvising jazz musicians is shaped by underlying patterns of coordination. We recorded dyads of professional jazz pianists improvising in two conditions of interaction: one in which they improvised together simultaneously (coupled), and an "overdubbed" (one-way) condition which precluded mutual adaptation because recordings were laid down sequentially. Interaction conditions enforced different causal networks which constrained the directed flow of musical information. Improvisers could mutually respond to one another in coupled trials but in one-way trials there was only one direction of causal influence (i.e from recording to live musician). These differing causal entanglements systematically altered the music produced by dyads, in terms of closely synchronized note onsets, response patterns in rhythmic activity level, and emergent tonal structure. These behavioral results were paralleled in the subjective experience of participants, who rated coupled trials as producing better music and facilitating easier and more effective coordination.

Interaction conditions were reflected in a lagged crosscorrelation analysis of co-performers' onset density time series (as well as a Granger causality analysis, reported in the SI). Co-performers' onset densities were more positively correlated in coupled trials. Within one-way trials, correlation was higher for ghost-to-live than live-to-ghost lags. These findings reveal a general tendency for musicians to match the activity level (i.e. onset density) of their partners, which occurred mutually in coupled trials but asymmetrically in one-way trials as ghost recordings were unresponsive to the onset density of live musicians. This echoes a related study on dyadic musical improvisation which applied Granger causality to loudness (correlated with onset density) time series of musician pairs 
playing with experimentally manipulated social attitudes (e.g. caring, dominant) (35). More generally, onset density matching brings to mind previous dyadic conversation studies, which have shown that people spontaneously entrain their movements, and mimic one another's facial expressions, manual gestures, eye gaze and acoustic speech characteristics when verbally interacting with one another $(26,37-40)$. Such behavioral matching has been proposed to facilitate successful communication by offloading predictive emulation (i.e. of a conversation partner's future behavior) onto one's own behavior (27), and this same interpersonal mechanism may underlie the onset density matching observed here.

A novel lagged consonance analysis was performed to quantify the directed flow of tonal information from one musician to another. This analysis revealed that musicians harmonize with the past notes of their partner, which occurs mutually in coupled trials but asymmetrically in one-way trials, as live musicians can harmonize with preceding notes of the live recording, but not vice versa. Whereas the previous analysis revealed a tendency for onset density matching, this analysis reveals complementary coordination. It is not that musicians simply match the notes of their partner, they play new notes that create consonant harmonies with one another. Matching and complementing are two important forms of coordination that co-occur in many JA contexts, as we see here. Taken together, this and the preceding onset density analyses build on previous ensemble performance studies which have shown that leadership and causal influence are reflected in the temporal coordination and postural sway of co-performing musicians $(14,17,35)$. Here we show it is possible to quantify the directed exchange of rhythmic and music theoretic tonal information in freely improvising musicians.

Our lagged consonance analysis was inspired by the technique of Cross Recurrence Quantification Analysis (CRQA), which has been used extensively in joint action research to study the dynamics of dyadic interaction $(5,37,38,41)$. In these applications, CRQA operates on similarity matrices quantifying behavioral matching of interacting individuals over time and across a range of time lags. CRQA has the flexibility to detect nonlinear patterns of coordination which can evolve over time, unlike related time series analyses such as Granger causality which assume stationary time series $(42,43)$. Our lagged consonance analysis is an extension of CRQA: instead of using a similarity measure to quantify behavioral matching, we used a measure of tonal consonance to quantify how co-performers harmonize their notes with one another. This allowed us to assess complementary coordination (as opposed to mimicry) with a functional, domain-specific operationalization. Complex, naturalistic human interaction often involves such complementary, functional coordination, and similar lagged analyses can be used with appropriate operationalizations of domain-specific coordination in future joint action research.

At the group-level, interaction condition shaped the emergence and dynamics of tonal structure. Mutual coupling enhanced pianists' tonal coordination, as they achieved greater Emergent Consonance (EC), which indicated an enhanced ability to harmonize their notes with one another. Moreover, EC systematically increased throughout improvised performances in both conditions, but significantly more so in coupled trials. This echoes previous findings that successful group coordination can emerge spontaneously, without explicit com- munication, as individuals interact and work together towards a common goal (44-46). Interestingly, in the case of improvised music, the dynamics of how coordination evolves throughout real-time performance are reflected in the music generated, and may be central to our aesthetic experience of collaboratively improvised music as listeners.

In sum, this study demonstrates that the music produced by improvising jazz ensembles, as well as the subjective impressions of performers, is shaped by underlying patterns of coordination. These findings have implications for the commonly practiced studio recording technique of "overdubbing" - which produces systematically different music than lively interaction, and we have documented some of those differences. Addtionally, our measures of coordination in expert improvisers can be incorporated in the design of generative and interactive AI music systems to make them more human-like and more musical $(11,22,47-50)$. This work also contributes to our understanding of JA more generally. The current results show that with appropriate operationalizations, it is possible to quantitatively assess how features of emergent group performance are constrained by and thus reflective of underlying, dynamic patterns of coordination.

\section{Materials and Methods}

Participants. 28 professional jazz pianists (25 male, 3 female) from the New York City music scene participated in this study. Participant age ranged from 21-37. On average participants had over 22 years experience playing piano $(\mathrm{sd}=5.2)$ and 15 years experience improvising $(\mathrm{sd}=4.6)$. All participants received formal training in piano performance and jazz studies at elite conservatories. None of our subjects had prior experience performing with one another.

Apparatus. Two MIDI-enabled keyboards were used: a Roland JunoDi and Nord Electro 2, both of which had 61 semi-weighted keys. Both keyboards were used on every trial (i.e. one-way trials were arranged such that the live pianist played whatever keyboard their ghost partner did not play). Ableton Live 9 Lite (running on a MacBook Air) was used to collect isolated MIDI recordings for each musician. Ableton was also used to synthesize the audio that participants heard, in the fashion of an electric Rhodes. This ensured time alignment of MIDI recordings, and that participants heard the same exact timbre for themselves and their partner, irrespective of condition. Participants were recorded at a music studio in Brooklyn, NY. The studio was divided by a curtain such that participants could not see one another. Participants heard themselves and their partners through Sony CH700N Noise Cancelling headphones. Thus, from participants' perspectives there was no visual or audible indication of their condition on a given trial.

Design and Procedure. Participants played a series of short (4-7 minute) 'free' improvisations, with no accompanying stimuli and no a priori musical template or constraints. Other than the suggested time frame, the only instruction participants were given was to do their best to improvise a compelling piece of music, as they would in a typical performance setting. Participants were informed of the two interaction conditions, but were not told which condition they were playing in on any given trial. After each trial, they responded to questionnaires indicating their subjective experience playing in the previous trial in terms of: (1) how easy it was to coordinate with their partner (2) how well coordinated they were with their partner (3) quality of the improvised piece and (4) degree to which they played a leader versus a supporter role.

Pairs of participants entered the studio in separate sessions. Each participant played at least 3 trials in both conditions, and played with the same 'live' partner for each of their coupled trials and the same 'ghost' partner for each of their one-way trials. Conditions were interleaved within sessions and counterbalanced across sessions 
to control for possible order effects. Individual tracks from coupled trials yoked one-way trials in the following session, as depicted in the SI. 50 coupled trials and 86 one-way pieces were recorded altogether. Pieces had an average duration of 342 seconds ( $\min =108$ seconds, $\max =738$ seconds, $\mathrm{sd}=12$ seconds). This dataset will be accessible on Github upon publication of the paper.

Tonal Consonance Measure. Our tonal consonance measure is based on the Tonal Spiral Array model, which has been validated against listener ratings and expert music theory analyses (31-33). Table 1 shows model ratings for exemplar pitch sets. See SI for specification of the measure.

Table 1. Consonance ratings of exemplar pitch sets.

\begin{tabular}{ll}
\hline Pitch Set & Consonance \\
\hline$\{C, E, G\}(C m a j)$ & .65 \\
$\{C, E b, G\}(C m i n)$ & .65 \\
$\{C, B, G\}$ & .54 \\
$\{C, E, G, F, A, C\}(C m a j+$ Fmaj) & .49 \\
$\{C, B\}$ & .48 \\
$\{C, E, G, F \#, A \#, C \#\}(C m a j+$ F\#maj) & .13 \\
serial (all 12 pitches) & .09 \\
\hline
\end{tabular}

ACKNOWLEDGMENTS. We are grateful to Douglas Hofstadter, Eduardo Izquierdo, Tyler Marghetis, Ashley Walton, David Landy, members of the Percepts and Concepts lab at Indiana University, and members of Todd Gureckis' Computation and Cognition Lab at New York University for their valuable support and feedback. Thanks also to all of the talented musicians who participated in this study.

1. Sebanz N, Bekkering H, Knoblich G (2006) Joint action: bodies and minds moving together. Trends in cognitive sciences 10(2):70-76.

2. Knoblich G, Butterfill S, Sebanz N (year?) Chapter three - psychological research on join action: Theory and data in Psychology of Learning and Motivation, Advances in Research and Theory, ed. Ross BH. (Academic Press) Vol. 54, pp. 59-101.

3. Hasson U, Ghazanfar AA, Galantucci B, Garrod S, Keysers C (2012) Brain-to-brain coupling: a mechanism for creating and sharing a social world. Trends in cognitive sciences 16(2):114121.

4. Goldstone RL, Gureckis TM (2009) Collective behavior. Topics in cognitive science 1(3):412438

5. Richardson DC, Dale R, Kirkham NZ (2007) The art of conversation is coordination. Psychological science 18(5):407-413.

6. Guimera R, Uzzi B, Spiro J, Amaral LAN (2005) Team assembly mechanisms determine collaboration network structure and team performance. Science 308(5722):697-702.

7. Council NR, , et al. (2015) Enhancing the effectiveness of team science. (National Academies Press)

8. Rebsamen B, et al. (2010) A brain controlled wheelchair to navigate in familiar environments. IEEE Transactions on Neural Systems and Rehabilitation Engineering 18(6):590-598.

9. D'Ausilio A, et al. (2012) Leadership in orchestra emerges from the causal relationships of movement kinematics. PLoS one 7(5):e35757.

10. Borgo D (2005) Sync or swarm: Improvising music in a complex age. (A\&C Black).

11. Hennig $\mathrm{H}$ (2014) Synchronization in human musical rhythms and mutually interacting complex systems. Proceedings of the National Academy of Sciences 111(36):12974-12979.

12. Keller PE (2014) Ensemble performance: Interpersonal alignment of musical expression. Expressiveness in music performance: Empirical approaches across styles and cultures pp. 260-282.

13. Rasch RA (1979) Synchronization in performed ensemble music. Acta Acustica united with Acustica 43(2):121-131.

14. Keller PE, Appel M (2010) Individual differences, auditory imagery, and the coordination of body movements and sounds in musical ensembles. Music Perception: An Interdisciplinary Journal 28(1):27-46.

15. Goebl W, Palmer C (2009) Synchronization of timing and motion among performing musicians. Music Perception: An Interdisciplinary Journal 26(5):427-438.

6. Demos AP, Carter DJ, Wanderley MM, Palmer C (2017) The unresponsive partner: roles of social status, auditory feedback, and animacy in coordination of joint music performance. Frontiers in psychology 8:149.

17. Chang A, Livingstone SR, Bosnyak DJ, Trainor LJ (2017) Body sway reflects leadership in joint music performance. Proceedings of the National Academy of Sciences p. 201617657.

18. Johnson-Laird PN (2002) How jazz musicians improvise. Music Perception: An Interdisciplinary Journal 19(3):415-442.

19. Pressing J (1988) Improvisation: methods and models. John A. Sloboda (Hg.): Generative processes in music, Oxford pp. 129-178.

20. Berkowitz A (2010) The improvising mind: Cognition and creativity in the musical moment. (Oxford University Press).
21. Limb CJ, Braun AR (2008) Neural substrates of spontaneous musical performance: An fmri study of jazz improvisation. PLoS one 3(2).

22. Gillick J, Roberts A, Engel J, Eck D, Bamman D (2019) Learning to groove with inverse sequence transformations. arXiv preprint arXiv:1905.06118.

23. Richardson MJ, Marsh KL, Isenhower RW, Goodman JR, Schmidt RC (2007) Rocking together: Dynamics of intentional and unintentional interpersonal coordination. Human movement science 26(6):867-891.

24. Hove MJ, Risen JL (2009) It's all in the timing: Interpersonal synchrony increases affiliation. Social cognition 27(6):949-960.

25. Valdesolo P, Ouyang J, DeSteno D (2010) The rhythm of joint action: Synchrony promotes cooperative ability. Journal of Experimental Social Psychology 46(4):693-695

26. Shockley K, Richardson DC, Dale R (2009) Conversation and coordinative structures. Topics in Cognitive Science 1(2):305-319.

27. Garrod S, Pickering MJ (2009) Joint action, interactive alignment, and dialog. Topics in Cog nitive Science 1(2):292-304.

28. Paulus PB, Levine DS, Brown V, Minai AA, Doboli S (2010) Modeling ideational creativity in groups: Connecting cognitive, neural, and computational approaches. Small Group Research 41(6):688-724.

29. Farbood MM (2012) A parametric, temporal model of musical tension. Music Perception: An Interdisciplinary Journal 29(4):387-428.

30. Johnson-Laird PN, Kang OE, Leong YC (2012) On musical dissonance. Music Perception An Interdisciplinary Journal 30(1):19-35

31. Chew $\mathrm{E}$, et al. (2014) Mathematical and computational modeling of tonality. AMC 10:12.

32. Chew $E$ (2005) Regards on two regards by messiaen: post-tonal music segmentation using pitch context distances in the spiral array. Journal of New Music Research 34(4):341-354.

33. Herremans D, Chew E, , et al. (2016) Tension ribbons: Quantifying and visualising tonal tension.

34. Walton AE, et al. (2018) Creating time: Social collaboration in music improvisation. Topics in cognitive science 10(1):95-119.

35. Aucouturier JJ, Canonne C (2017) Musical friends and foes: The social cognition of affiliation and control in improvised interactions. Cognition 161:94-108.

36. Kirschner S, Tomasello M (2009) Joint drumming: social context facilitates synchronization in preschool children. Journal of experimental child psychology 102(3):299-314

37. Louwerse MM, Dale R, Bard EG, Jeuniaux P (2012) Behavior matching in multimodal communication is synchronized. Cognitive science 36(8):1404-1426.

38. Richardson DC, Dale R (2005) Looking to understand: The coupling between speakers' and isteners' eye movements and its relationship to discourse comprehension. Cognitive science 29(6):1045-1060.

39. Shockley K, Santana MV, Fowler CA (2003) Mutual interpersonal postural constraints are involved in cooperative conversation. Journal of Experimental Psychology: Human Perception and Performance 29(2):326.

40. Abney DH, Paxton A, Dale R, Kello CT (2014) Complexity matching in dyadic conversation. Journal of Experimental Psychology: General 143(6):2304.

41. Dale R, Spivey MJ (2006) Unraveling the dyad: Using recurrence analysis to explore patterns of syntactic coordination between children and caregivers in conversation. Language Learning 56(3):391-430.

42. Marwan N, Romano MC, Thiel M, Kurths J (2007) Recurrence plots for the analysis of complex systems. Physics reports 438(5-6):237-329.

43. Barnett L, Seth AK (2014) The mvgc multivariate granger causality toolbox: a new approach to granger-causal inference. Journal of neuroscience methods 223:50-68.

44. Roberts ME, Goldstone RL (2011) Adaptive group coordination and role differentiation. PLoS One 6(7).

45. Andrade-Lotero E, Goldstone RL (year?) Self-organized division of cognitive labor.

46. Heylighen $F(2013)$ Self-organization in communicating groups: the emergence of coordination, shared references and collective intelligence in Complexity Perspectives on Language, Communication and Society. (Springer), pp. 117-149.

47. Hennig $\mathrm{H}$, et al. (2011) The nature and perception of fluctuations in human musical rhythms. Plos one 6(10):e26457.

48. Roberts A, et al. (2019) Approachable music composition with machine learning at scale in Proceedings of the 20th International Society for Music Information Retrieval Conference (ISMIR).

49. Huang CZA, et al. (2019) Music transformer: Generating music with long-term structure.

50. Hawthorne C, et al. (2019) Enabling factorized piano music modeling and generation with the maestro dataset. 


\section{Supplementary Information for}

2 Professional improvising musicians couple more closely in timing and tonality when simultaneously rather than successively recording

4 Matt Setzler, Robert Goldstone

Matt Setzler

E-mail: msetzler@indiana.edu

7 This PDF file includes:

Supplementary text

Figs. S1 to S12

Tables S1 to S4

SI References 


\section{Supporting Information Text \\ Tonal Consonance Model}

Our measure of tonal consonance was adapted from a previous model of musical tonality called the Tonal Spiral Array, which has been validated against listener ratings and expert music theory analyses of musical tension $(1,2)$. While there are alternative models of tonal consonance to consider $(3,4)$, the Tonal Spiral Array is especially appropriate in the context of freely improvised music because has been validated with respect to both functional and post-tonal music (5), and has the flexibility to model temporally extended (as opposed to just instantaneous) tonal structure (1). We adapted the "cloud diameter" measure of tonal dissonance (2) to create conditions of enharmonic equivalence (e.g. D\# and Eb are treated equivalently in our measure).

The rationale behind the consonance measure is that certain pairwise pitch intervals are inherently more or less consonant. For example, a tritone (e.g. $\{\mathrm{C}, \mathrm{F} \#\}$ ) is highly dissonant (lowly consonant), whereas a perfect 5 th (e.g. $\{\mathrm{C}, \mathrm{G}\}$ ) is highly consonant (lowly dissonant). Accordingly, every interval class was assigned a 'dissonance rating', as indicated in Figure S2A (2). These dissonance ratings were taken from previous applications of the Tonal Spiral Array $(2,5)$, as the closest euclidean distance between pitch pairs for each interval. Tonal consonance was computed for a given window of music as the negative weighted sum of dissonance ratings, scaled by the normalized frequency at which intervals occurred within said window (i.e. such frequencies of all interval classes summed to 1). In scaling by normalized frequency we ensured that there was no correlation between consonance and the amount of sustained sound within a window. Details of how normalized frequency distributions were obtained are depicted in Figure S3. Lastly, a constant of 2 was added to avoid negative values, since consonance is the opposite of dissonance. Table S1 shows model ratings for exemplar pitch sets.

Table S1. Consonance ratings of exemplar pitch sets.

\begin{tabular}{ll}
\hline Pitch Set & Consonance \\
\hline$\{C, E, G\}($ Cmaj) & .65 \\
$\{C, E b, G\}(C m i n)$ & .65 \\
$\{C, B, G\}$ & .54 \\
$\{C, E, G, F, A, C\}(C m a j+$ Fmaj) & .49 \\
$\{C, B\}$ & .48 \\
$\{C, E, G, F \#, A \#, C \#\}(C m a j+$ F\#maj) & .13 \\
serial (all 12 pitches) & .09 \\
\hline
\end{tabular}

Consonance time series were computed from music streams using a 5 second sliding window* with 0.2 second hop size, as illustrated in Figure S2B. Three measures of consonance were considered: Individual Consonance (consonance of individual music streams), Combined Consonance (consonance of merged music streams from both players in a dyad) and Emergent Consonance (Combined Consonance minus average Individual Consonance of both musicians in a dyad). Emergent consonance is essentially a measure of tonal coordination, as it captures the consonance arising from the interaction of pitches played by the two different musicians. A situation in which each pianist plays consonant notes that clash with one another would result in low emergent consonance (e.g. $\{\mathrm{C}, \mathrm{E}, \mathrm{G}\}$ and $\{\mathrm{F} \#, \mathrm{~A} \#, \mathrm{C} \#\}$ are consonant on there own but $\{\mathrm{C}, \mathrm{E}, \mathrm{G}, \mathrm{F} \#, \mathrm{~A} \#, \mathrm{C} \#\}$ is highly dissonant), whereas a situation in which each pianist plays dissonant pitch sets that stabilize one another when sounded together would result in high emergent consonance (e.g. $\{\mathrm{C}, \mathrm{B}\}$ and $\{\mathrm{E}, \mathrm{G}\}$ have low average consonance but $\{\mathrm{C}, \mathrm{E}, \mathrm{G}, \mathrm{B}\}$ has high consonance because it is tonicized to a Cmaj7 chord).

\section{SI Results}

Subjective Responses. At the conclusion of every trial participants filled out a questionnaire indicating their subjective experience of (1) quality of the improvised piece (2) how well coordinated they were with their partners (3) how easy it was to coordinate with their partners and (4) the degree to which they felt they played a leader or a supporter role. Responses to questions (1-3) were given on a 5 point Likert scale and responses to question 4 were given on a 5 point scale in which 1 corresponded to a strong leader role and 5 corresponded to a strong supporter role. Responses are depicted in Figure S4.

Cross-correlation of co-performer onset density. Cross-correlation of onset density decreases for large-magnitude lags, as depicted in Figure S5.

Relationship between onset density and tonal consonance. Although our measure of tonal consonance normalized for the combined duration of sustained notes, it did not explicitly normalize for onset density. Accordingly, we examined the correlation between consonance and onset densit in individual music streams. The relationship between these measures is depicted in Figure S6. Overall these plots indicate a negative correlation between consonance and onset density (correlation $=.377,95 \%$ $\mathrm{CI}=[.37, .38], \mathrm{t}(299466)=222.73, \mathrm{p}<0.01)$, although there is considerable spread of consonance especially at low levels of onset density which are more common in our dataset. Such a negative correlation is unsuprising and in fact a desired property: in time windows with more notes played (i.e. higher onset density) there are more opportunities for dissonant intervals to occur,

*All reported analyses were also conducted with 2 and 10 second window sizes and yielded the same results. 
resulting in more dissonance probabilistically. Nonetheless, this correlation made it necessary to deconfound effects of tonal consonance from those of onset density, as reported in subsequent supplementary analyses.

Granger Causality Between Co-Performers' Onset Density and Tonal Consonance. The Multivariate Granger Causality (MVGC) toolbox in MATLAB was used to compute Granger Causality (GC) amongst co-performers' onset density and tonal consonance time series (6). This toolbox allowed us to deconfound the correlation between onset density and tonal consonance by separately computing pairwise GC between onset density (conditioned on tonal consonance) and consonance (conditioned on onset density).

Data pre-processing and granger causality computation. Onset density and Consonance time series were obtained from each individual MIDI time series with 2 second windows sliding windows and 0.6 second step sizes. These time series were detrended with first differencing to remove non-stationarity. We had to account for missing values in Tonal Consonance time series, because Consonance is undefined in time windows with no playing. Accordingly, we subtracting the temporal mean of each consonance time series from every value, and set missing values to 0 .

Once the data was pre-processed, pairwise GC between co-players' Onset Density and Consonance time series was computed separately for each trial, following the procedures of the MVGC toolbox demo. This included a test to ensure time series passed the Granger causality stationarity assumption, with spectral radius of less than one. This condition was met by time series in all trials except for 2, which were discarded from further analysis.

GC computation involves a model comparison between a restricted Vector Autoregressive Model (VAR) (e.g. predict A from A's past) with a full VAR model (e.g. predict A from A's past and B's past). As suggested in the literature (6), these should each have the same model order. GC was computed repeatedly for each trial over a range of hand-chosen model orders. Since our time series came from sliding windows, VAR models with small orders would be fit with data in which there is substantial temporal overlap between predictors and predicted values. We avoided this issue by hand-choosing reasonable model orders that were sufficiently high to avoid such temporal overlap. Moreover, in computing GC for each trial over a range of model orders we were able to see how robust GC results were to model order.

Results and Analysis. GC was compared across three conditions: ghost-to-live (GC from ghost recording to live musicians in one-way trials), live-to-ghost (vise versa) and live-to-live (granger causality between mutually interacting musicians in coupled trials). The main GC output was a log-likelihood model comparison of full and restricted models, which we used as a continuous dependent measure of causality as has been done in previous applications (7). Results for are depicted in Figure S7.

Overall there is higher GC of onset density compared to tonal consonance. GC of onset density reflects the underlying patterns of influence enforced by each condition. Within one-way trials, GC of ghost-to-live was higher than live-to-ghost (paired $\mathrm{t}(84)=3.724, \mathrm{p}<0.01$; comparing gc values in each direction within each one-way trial, and using GC values obtained with 5.4 second model order). Thus, onset density of live musicians in one-way trials was responsive to that of the ghost recordings they were playing with but not the other way around. There was no significant difference in GC between ghost-to-live and live-to-live (i.e. coupled) conditions $(\mathrm{t}(283)=.101 ; \mathrm{p}=.92 ; 5.4$ second model order).

Granger causality has been applied in related work to show that leader follower relations in music ensembles playing composed music are reflected in the postural sway of performers $(7,8)$. Here we find a related to collectively improvised musical structure: experimentally manipulated conditions of interaction constrain the directed flow of musical information (such as onset density) within dyads of improvising pianists, and these different patterns of causal influence can be detected with Granger causality.

Lagged Consonance. In the main body of this paper we report that within one-way trials Emergent Consonance is higher when combining past notes of the ghost recording with future notes of live musician (ghost-to-live) as opposed to the other way around (live-to-ghost). This result was robust over the full range of window sizes, and the same results was also obtained with respect to Combined Consonance, as depicted in Figure S10. Results of supporting statistical tests are summarized below in Table S2.

\begin{tabular}{ccccc}
\hline Direction & Window Size $(\mathrm{sec})$ & $\mathrm{t}$ & $\mathrm{df}$ & $\mathrm{p}$-value \\
\hline Emergent Consonance & & & & \\
\hline ghost-live vs live-ghost & 2 & 2.6 & 85 & .012 \\
ghost-live vs live-ghost & 5 & 2.3 & 85 & .025 \\
ghost-live vs live-ghost & 10 & 2.4 & 85 & .019 \\
\hline Combined Consonance & & & & \\
\hline ghost-live vs live-ghost & 2 & 2.8 & 85 & .0067 \\
ghost-live vs live-ghost & 5 & 2.5 & 85 & .014 \\
ghost-live vs live-ghost & 10 & 2.4 & 85 & .016 \\
\hline Total Onset Density & & & & \\
\hline ghost-live vs live-ghost & 2 & 1.197 & 85 & .235 \\
\hline
\end{tabular}

Table S2. Results summary from lagged consonance analysis. Results are from a paired t-test comparing average consonance at positive lags (ghost-to-live) to negative lags (live-to-ghost) within each one-way trial. Lags ranged from +/- 20 seconds in increments of 2 seconds. 
Given the correlation between tonal consonance and onset density, we performed a supplementary analysis to confirm that these results are not somehow artifactual of condition-wise differences in onset density. The same lagged analysis was performed with respect to combined onset density instead of combined/emergent consonance. If onset density was driving the asymmetry in one-way trials we would expect to see a similar asymmetry in one-way with onset density (though in the opposite direction, because of the negative correlation). There was no such asymmetry (paired $\mathrm{t}(85)=1.197, \mathrm{p}=0.235$ ), indicating that the above results are genuinely reflective of tonal coordination.

Hierarchical Bayesian Model of Emergent Consonance Over Time. We were interested in how Emergent Consonance (EC) evolves over improvised pieces as a function of interaction condition. Since pieces were of different lengths we looked at EC over normalized time by averaging EC in equispaced time bins within each piece. This was done with a range of different bin sizes to ensure results weren't artifacts of a particular binning scheme, as depicted in Figure S11. These plots indicate systematic structure to how EC evolves over time which is robust across binning schemes. Overall there appears to be a curvilinear relationship: EC generally increases throughout pieces, and decreases slightly in the last $10 \%$. These plots also indicate an interaction between time and condition: EC appears to increase more in coupled trials.

Hierarchical Bayesian modeling was used to model EC over time. We used the rjags package (9) to estimate posterior distributions using Markov Chain Monte Carlo (MCMC) sampling, and followed best practices as described in (10). Given the curvilinear trend, EC was modeled as a quadratic function of time. Each piece was fitted with its own coefficients for linear and quadratic terms (and associated sigma parameters for variance) relating EC as a function of time. Piece-level parameters were modeled as being drawn from separate normal distributions for each condition-parameter combination. Parameters for condition-level distributions were modeled as being drawn from uniform prior distributions $([-2,2]$ for means and $[0,50]$ for sigmas). This approach allowed us to evaluate effects of interaction condition by comparing posterior estimates for means of the condition-level distributions. For ease of interpretation, condition-wise differences between posterior estimates of linear and quadratic terms were explicitly recorded throughout MCMC sampling (coupled minus one-way). This hierarchical model was fit repeatedly over a range of EC window sizes and time binnings to ensure results were not skewed by a particular window size of binning resolution, as depicted in Figure S12. Posterior estimates confirmed the aforementioned temporal structure suggested by Figure S11. Overall they indicate a negative quadratic trend (i.e. inverted U-shape) and a positive linear trend for both conditions. Importantly, the linear term was significantly more positive in coupled as opposed to overdubbed trials, indicating the EC increases more sharply in coupled trials. These results are presented in more detail for particular window sizes and binning schemes in the following subsections.

Hierarchical Bayesian model with 5 second window and 50 time bins. Posterior estimates from this particular binning scheme are presented in Table S3. The $95 \%$ confidence interval of the condition-wise difference in means for sampling distributions for the linear term is positive and non-inclusive of zero, indicating that there is a greater linear in increase of EC in coupled versus one-way trials.

\begin{tabular}{cccccc} 
Parameter & Average & SD & $95 \% \mathrm{Cl}$ & ESS & Gelman-Rubin Stat (Upper C.I.) \\
\hline linear; coupled & $6.358 \mathrm{e}-3$ & $8.784 \mathrm{e}-04$ & {$[4.633 \mathrm{e}-3,8.079 \mathrm{e}-3]$} & 7889.804 & 1.00 \\
linear; oneway & $4.182 \mathrm{e}-3$ & $6.723 \mathrm{e}-04$ & {$[2.867 \mathrm{e}-3,5.504 \mathrm{e}-3]$} & 8208.707 & 1.00 \\
linear; coupled minus oneway & $2.176 \mathrm{e}-03$ & $1.105 \mathrm{e}-03$ & {$[1.550 \mathrm{e}-06,4.340 \mathrm{e}-03]$} & 8171.120 & 1.00 \\
quadratic; coupled & $-1.095 \mathrm{e}-4$ & $1.692 \mathrm{e}-05$ & {$[-1.429 \mathrm{e}-4,-7.647 \mathrm{e}-5]$} & 9169.990 & 1.00 \\
quadratic; oneway & $-7.700 \mathrm{e}-5$ & $1.312 \mathrm{e}-05$ & {$[-1.030 \mathrm{e}-4,-5.148 \mathrm{e}-5]$} & 8220.254 & 1.00 \\
quadratic; coupled minus oneway & $-3.254 \mathrm{e}-05$ & $2.144 \mathrm{e}-05$ & {$[-7.471 \mathrm{e}-05,9.514 \mathrm{e}-06]$} & 8615.624 & 1.00 \\
\hline
\end{tabular}

Table S3. Posterior Estimates for model fit with data from 5 second sliding window, split into 50 time bins. Effective Sample Size (ESS) and Gelman-Rubin Stat are included as metrics of convergence and representativeness in the MCMC sampling.

Hierarchical Bayesian model with $\mathbf{5}$ second window and $\mathbf{2 0}$ time bins. Posterior estimates from this particular binning scheme are presented in Table S4. Evidence Ratio for hypothesis that linear trend is more positive in coupled trials is 150.5. Evidence Ratio for hypothesis that quadratic trend is more negative in coupled trials is 59.3

\begin{tabular}{cccccc} 
Parameter & Average & SD & $95 \% \mathrm{Cl}$ & ESS & Gelman-Rubin Stat (Upper C.I.) \\
\hline linear; coupled & $1.55 \mathrm{e}-2$ & $1.55 \mathrm{e}-3$ & {$[1.24 \mathrm{e}-2,1.8 \mathrm{e}-2]$} & 1258.8 & 1.01 \\
linear; oneway & $1.05 \mathrm{e}-2$ & $1.193 \mathrm{e}-3$ & {$[8.17 \mathrm{e}-3,1.28 \mathrm{e}-2]$} & 1315.5 & 1.00 \\
linear; coupled minus oneway & 0.0049137 & $1.961 \mathrm{e}-03$ & {$[1.0395 \mathrm{e}-3,8.7168 \mathrm{e}-3]$} & 1224.668 & 1.01 \\
quadratic; coupled & $-6.491 \mathrm{e}-4$ & $-7.079 \mathrm{e}-5$ & {$[-7.865 \mathrm{e}-4,-5.086 \mathrm{e}-4]$} & 1239.531 & 1.01 \\
quadratic; oneway & $-4.553 \mathrm{e}-4$ & $-5.563 \mathrm{e}-5$ & {$[-5.639 \mathrm{e}-4,-3.448 \mathrm{e}-4]$} & 1267.477 & 1.00 \\
quadratic; coupled minus oneway & -0.0001938 & $9.017 \mathrm{e}-05$ & {$[-3.695 \mathrm{e}-4,-1.53 \mathrm{e}-5]$} & 1179.448 & 1.01 \\
\hline
\end{tabular}

Table S4. Posterior Estimates for model fit with data from 5 second sliding window, split into 20 time bins. Effective Sample Size (ESS) and Gelman-Rubin Stat are included as metrics of convergence and representativeness in the MCMC sampling. 
Tests for Artifacts of Onset Density. Two supplementary analyses were performed to verify that these results were not artifactual of onset density. First we looked for correlation between onset density (combined within a dyad) and Emergent Consonance. As is displayed in Figure S8, there is no strong correlation between these features, except for extremely high values of onset density which were outliers. Second, we contrasted overall level of individual consonance between musicians playing in coupled and one-way trials. If EC results were artifactual of there being more onset density in coupled trials, we would expect lower individual consonance in coupled trials which would in turn result in higher EC. But, as depicted in Figure S9 this is not the case. 

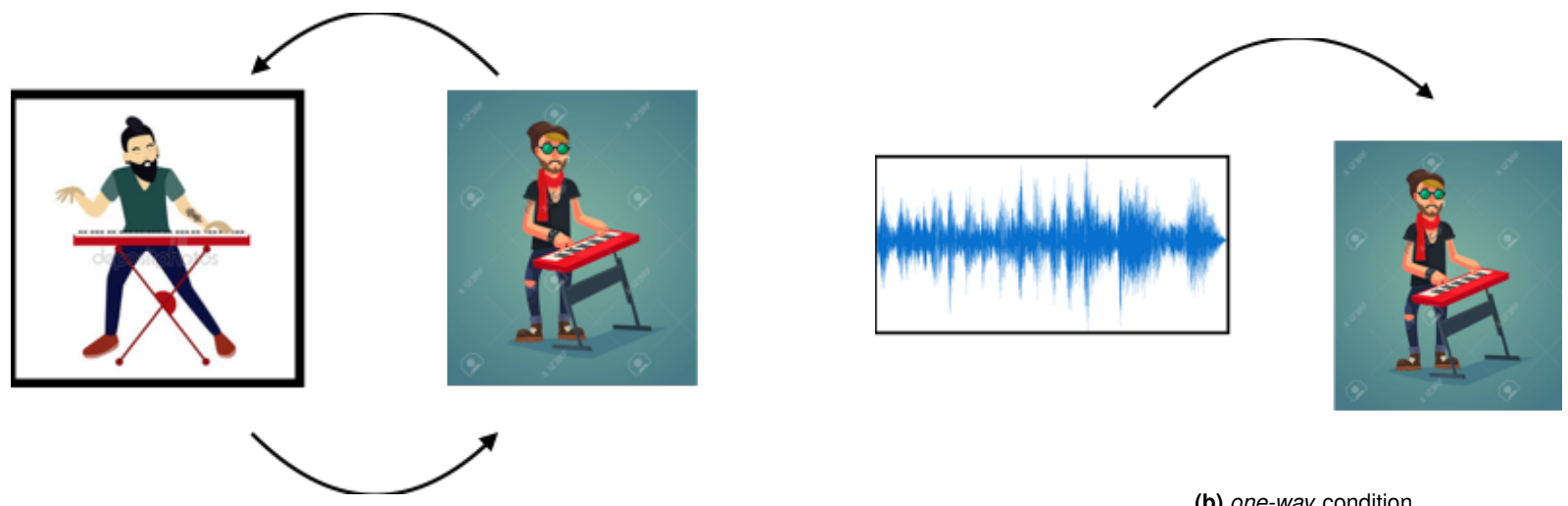

(a) coupled condition

(b) one-way condition

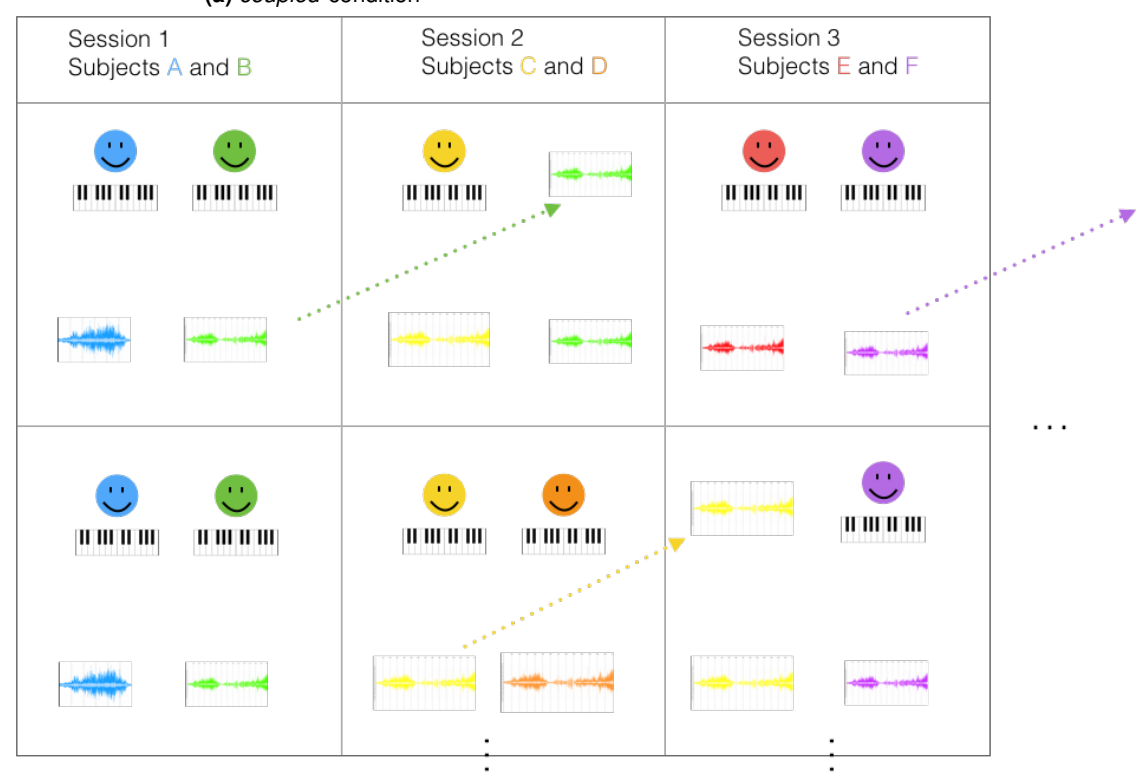

(c) Yoked experimental design. Pairs of participants entered the studio in separate sessions. Each participant played with the same live partner for all coupled trials and the same ghost partner for all one-way trials. Participants played at least 3 trials in each condition. Conditions were interleaved within sessions and counterbalanced across sessions to control for possible order effects. Individual tracks from each coupled trial were used to yoke one-way trials in the following session.

Fig. S1. Experimental procedure. 


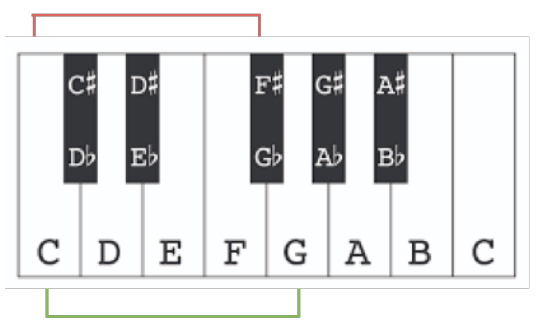

(a)

\begin{tabular}{|c|c|}
\hline interval & dissonance \\
\hline unison & 1.0 \\
\hline m2/M7 & 2.6 \\
\hline M2/m6 & 2.2 \\
\hline m3/M6 & 1.9 \\
\hline M3/m6 & 1.7 \\
\hline P4/P5 & 1.5 \\
\hline A4 & 3.2 \\
\hline
\end{tabular}

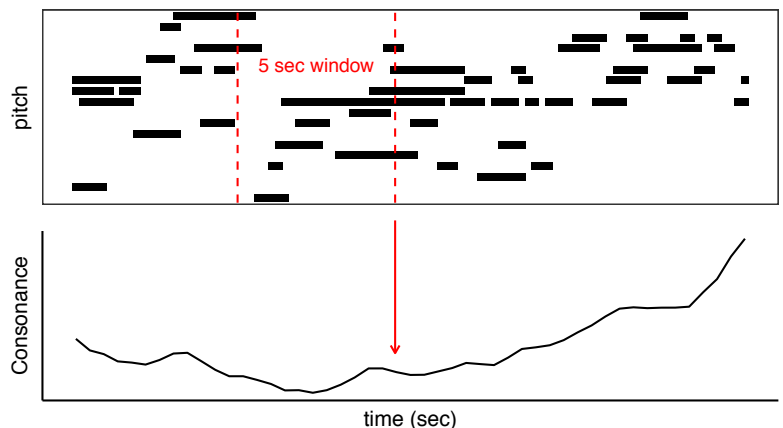

(b)

Fig. S2. Tonal consonance measure. (a) Every pitch interval is assigned a dissonance rating (perfect fifth and tritone are colored for illustration). Tonal consonance is the negative weighted sum of dissonance levels scaled by how often intervals occur within windows of music. (b) Consonance time series were computed from music sequences using 5 second sliding windows. 


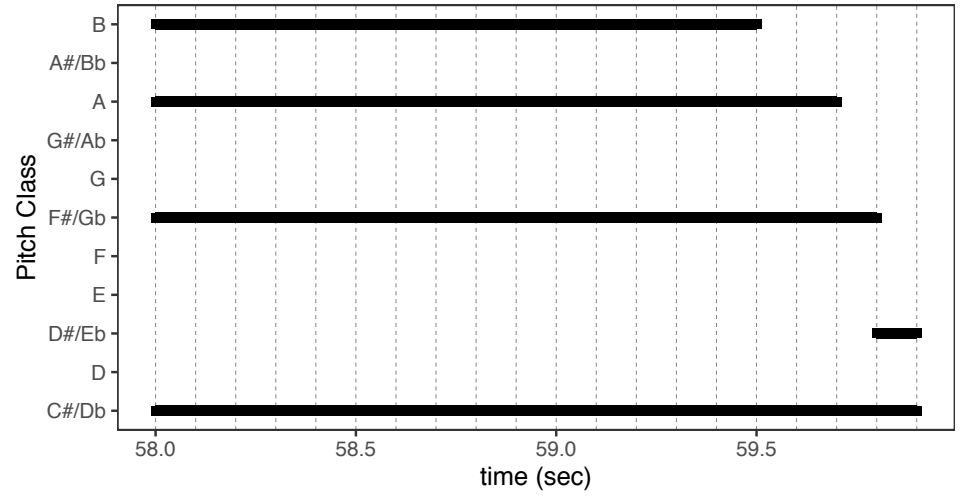

(a)

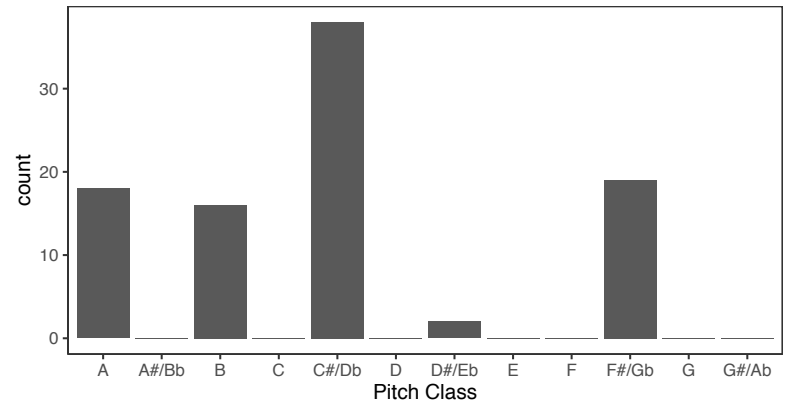

(b)

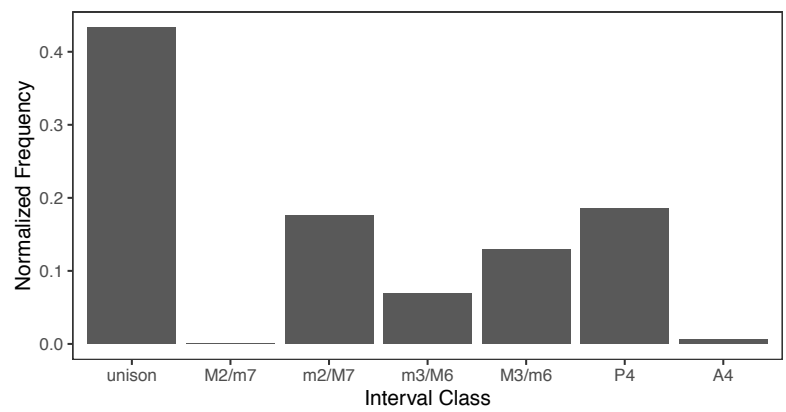

(c)

Fig. S3. Tonal consonance measure for arbitrary 2 second window of music. Frequency distributions for every pitch class (b) were obtained by splitting music windows into 200 millisecond bins and counting the number of bins where each piece class was active (a). Pitch class distributions were then used to determined normalized frequency distributions of intervals (c), by determining how much each interval class was active and normalizing such that frequencies of all intervals summed to 1. 

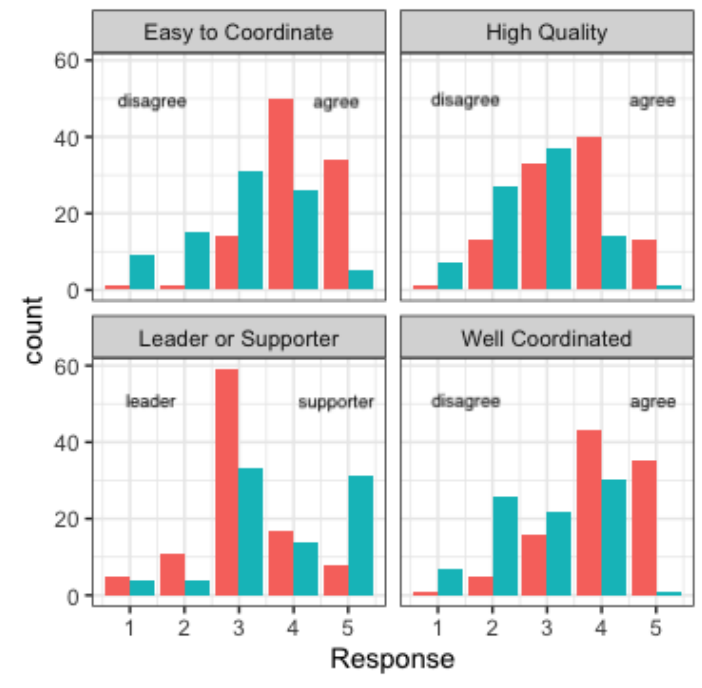

condition one-way

(a)
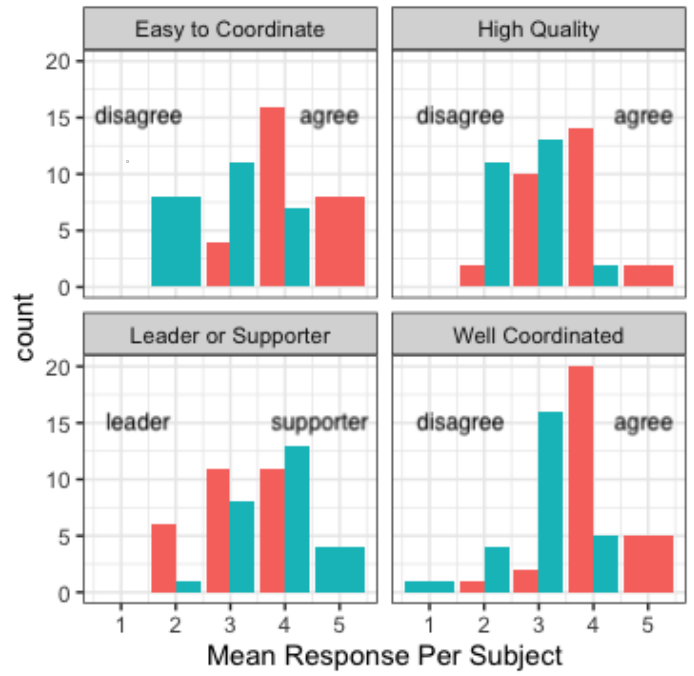

condition coupled one-way

(b)

Fig. S4. Subjective responses. (A) depicts counts of all participant responses across each condition and (B) depicts counts of the average response of every participant within each condition. 


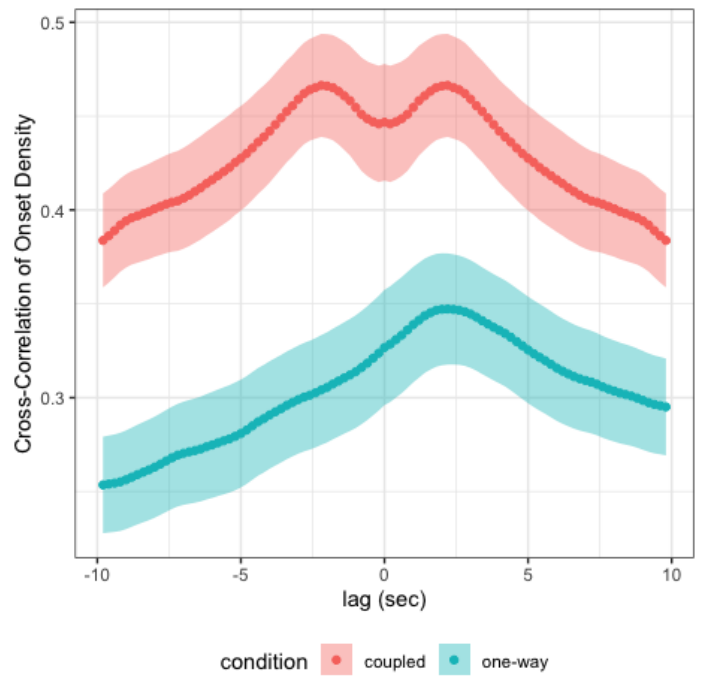

(a)

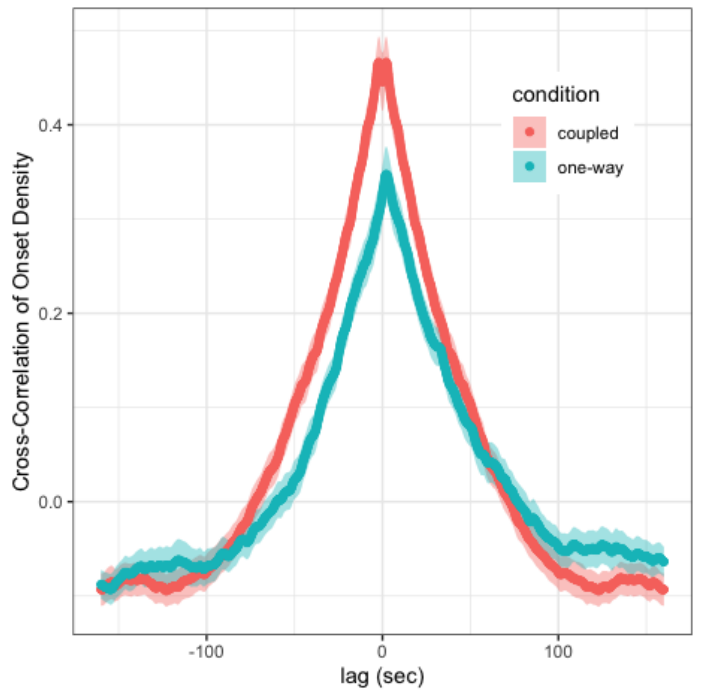

(b)

Fig. S5. Cross-correlation (cc) of onset density between co-performers. Onset density was computed for individual music streams with a 2 second sliding window and 0.2 second hop size. (a) CC is higher overall in coupled trials. Also, within one-way trials cc is higher at positive lags (ghost-to-live) as opposed to negative lags (live-to-ghost), indicating that onset density of live performers responds to that of ghost recordings but not the other way around. (b) CC in two conditions converge at lags of large magnitude. 


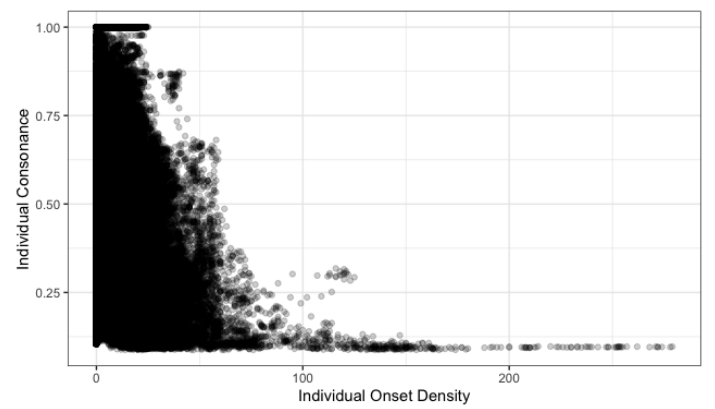

(a)

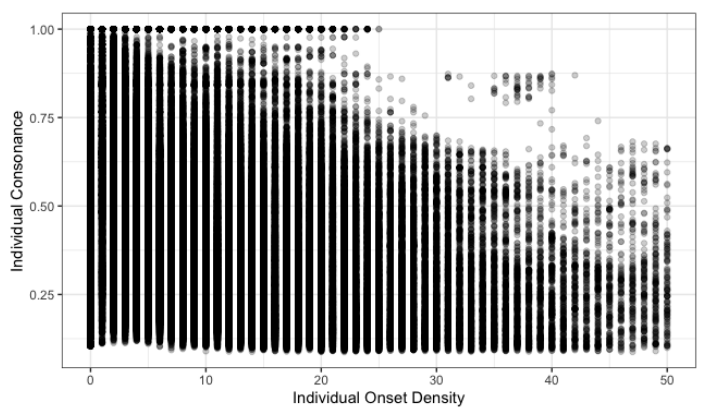

(b)

Fig. S6. Relationship between tonal consonance and onset density in individual music streams. Each point represents onset density and tonal consonance values at simultaneous time indices within individual music streams. 


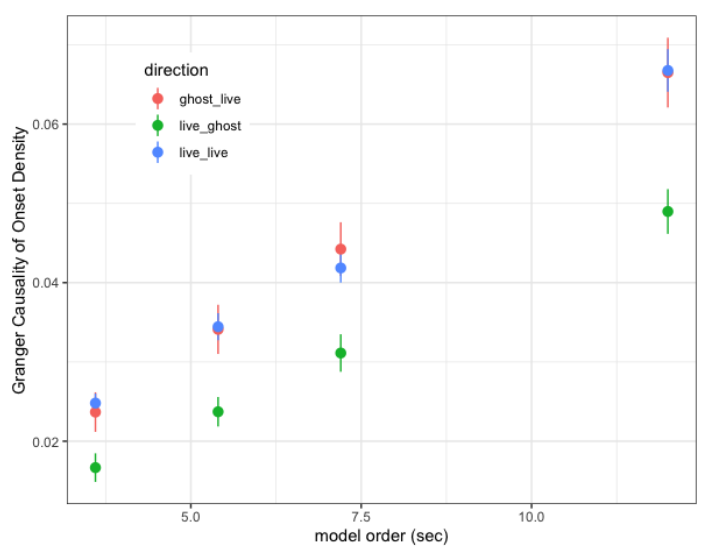

(a)

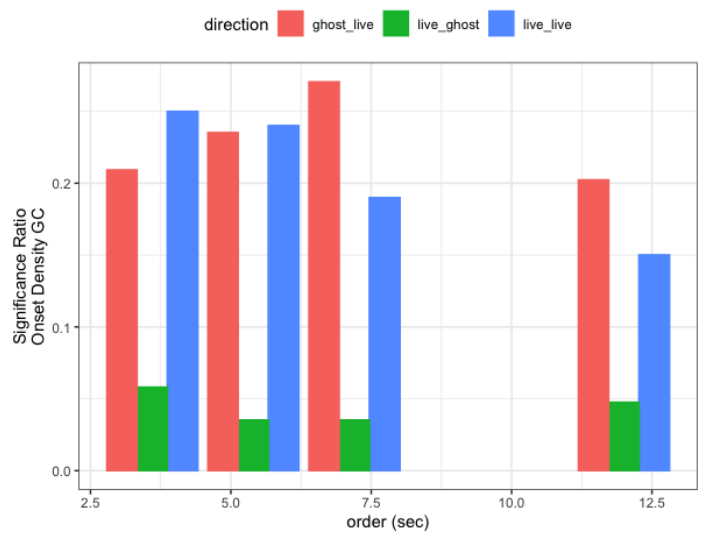

(c)

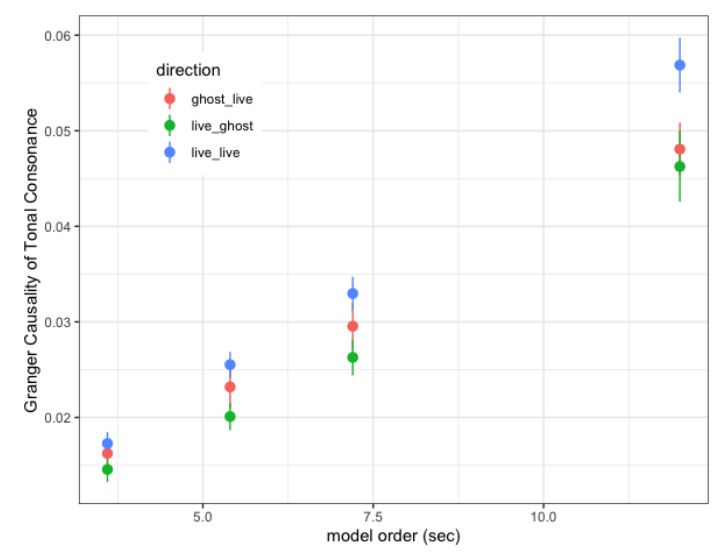

(b)

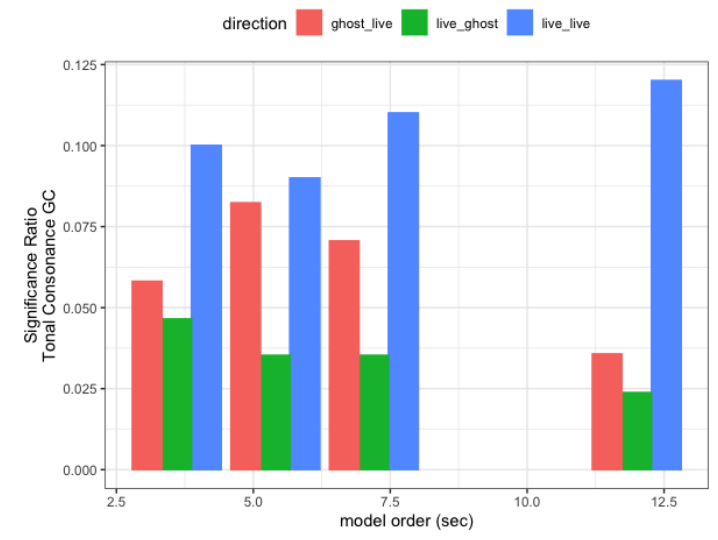

(d)

Fig. S7. Granger causality reflects directed flow of improvised musical information. (A) and (B) depict mean GC of co-performer onset density and tonal consonance, respectively, for all trials within each condition computed across a range of model orders ( $x$-axis). Error bars denote standard error. (C) and (D) depict the proportion of trials in which GC was statistically significant at 0.05 significance levels. 


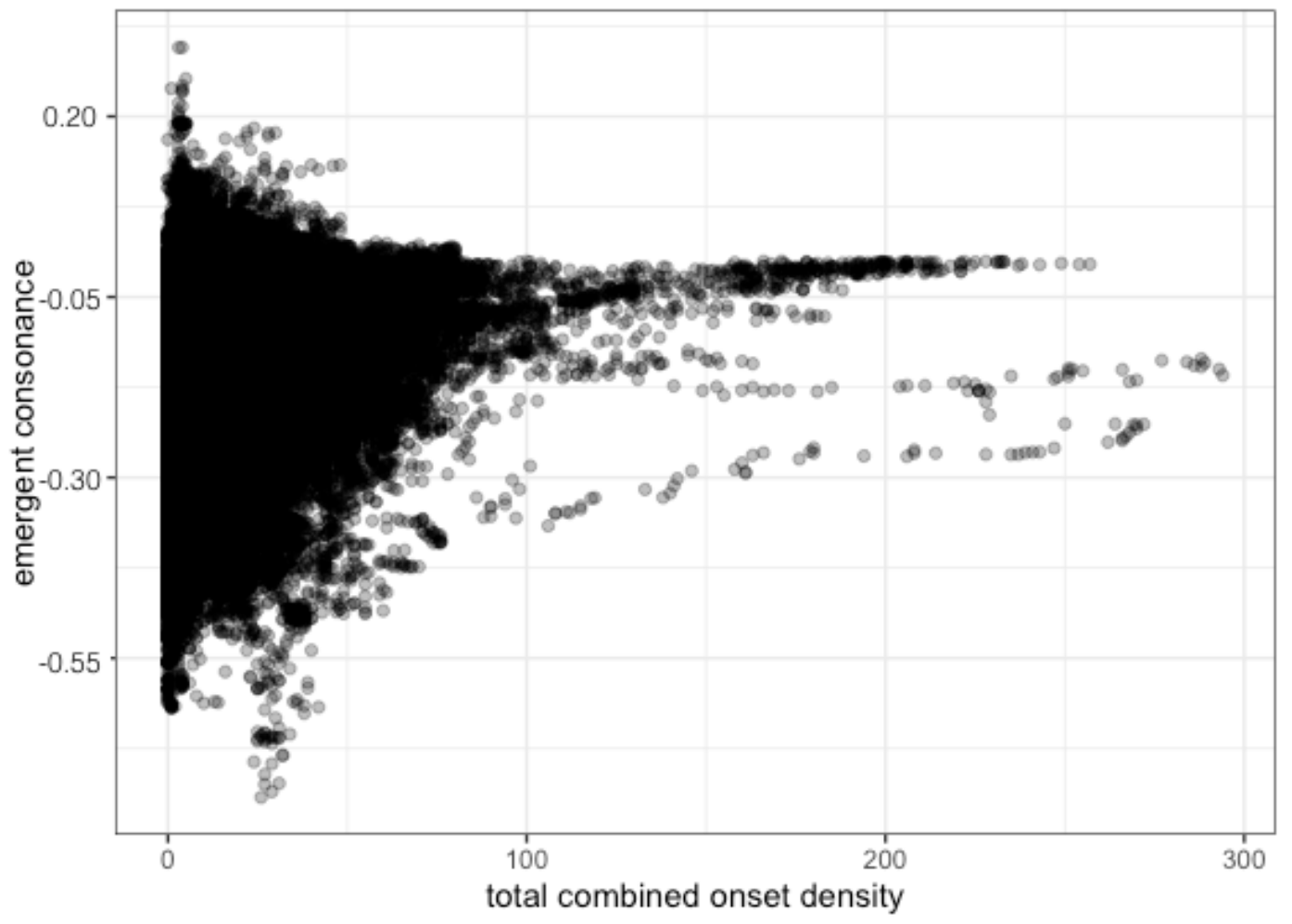

Fig. S8. Combined onset density and Emergent Consonance (EC). Each point depicts combined onset density (2 second sliding window with 0.2 second hop size) and EC at simultaneous time indices across all pieces. For the most part there is no correlation between onset density and emergent consonance, except for outliers of extremely high onset density. 


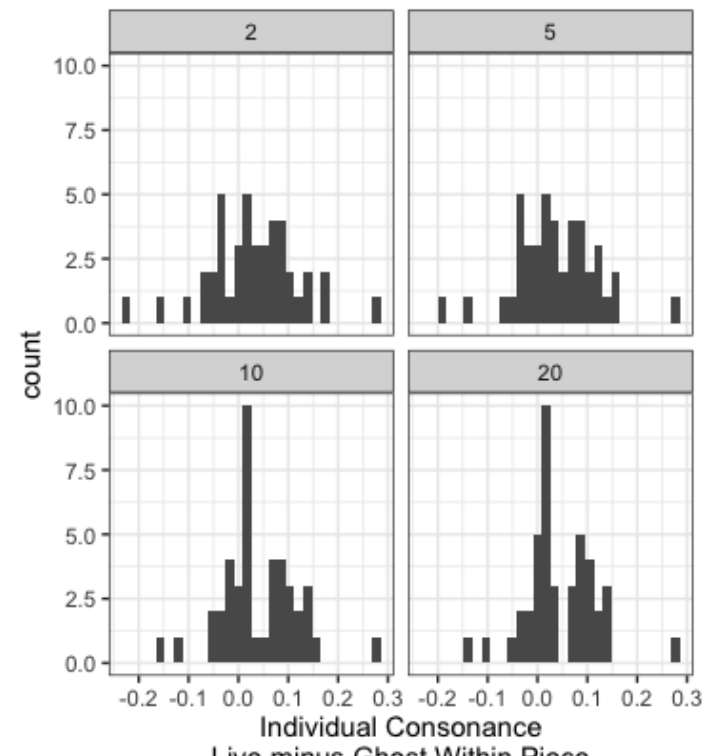

Live minus Ghost Within Piece

(a)

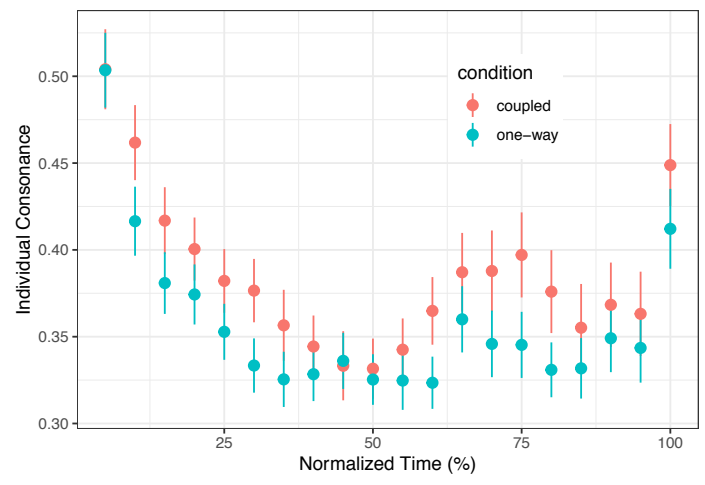

(b)

Fig. S9. Individual tonal consonance over time in coupled and one-way trials. (A) depicts the difference between average individual tonal consonance throughout coupled pieces minus that of the correspondingly yoked one-way pieces. This resulted in one value (coupled - one-way) for each yoked ID for every window size of tonal consonance (2, 5,10 and 20 seconds all with 0.2 second hop size). Distributions are depicted for each window size. (B) Depicts individual tonal consonance (5 second sliding window) over normalized time. Time was normalized by dividing each piece into 20 equispaced temporal bins and averaging individual consonance within each bin. Points display mean consonance across all pieces within each condition and error bars denote standard error of the mean. 


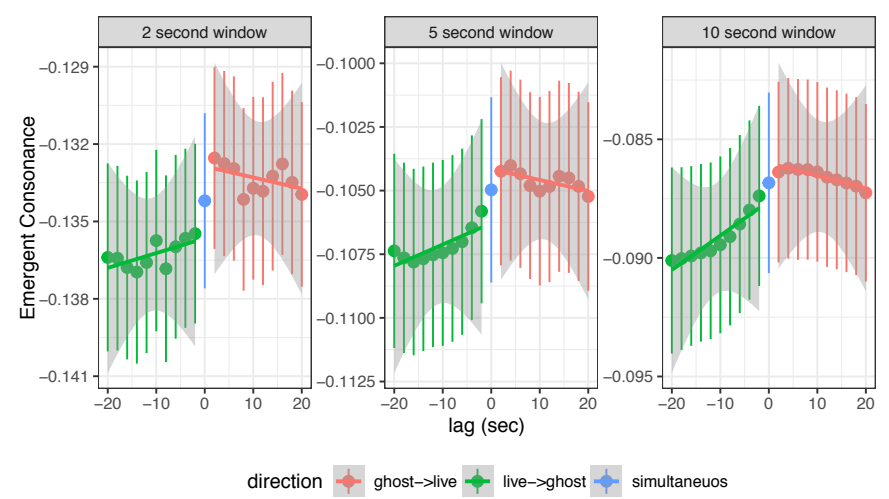

(a) Lagged emergent consonance in one-way trials.

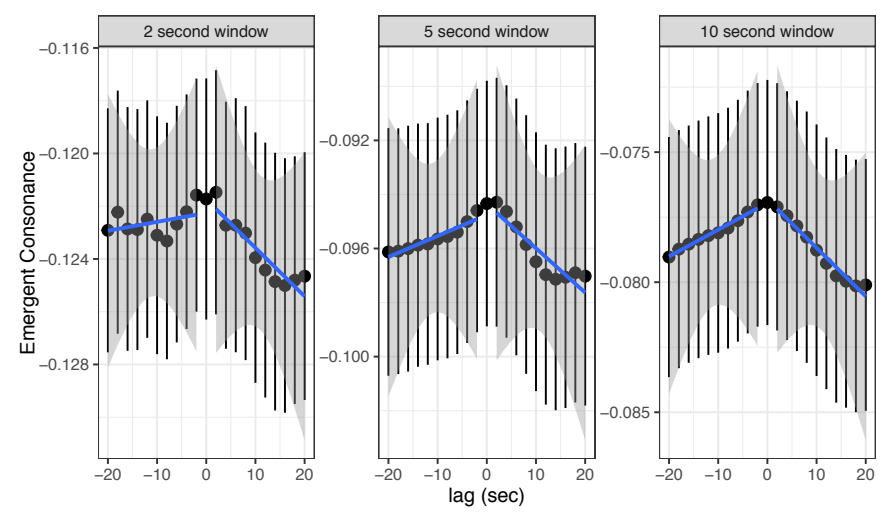

(b) Lagged emergent consonance in coupled trials.

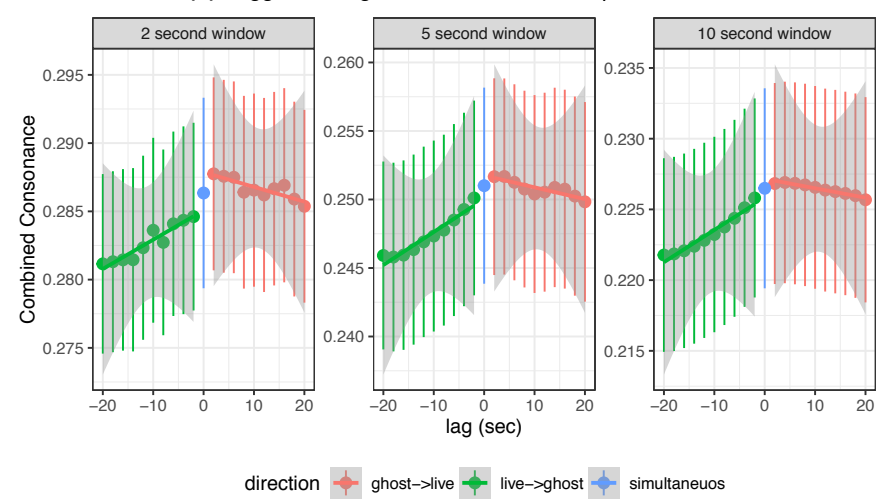

(c) Lagged combined consonance in one-way trials.

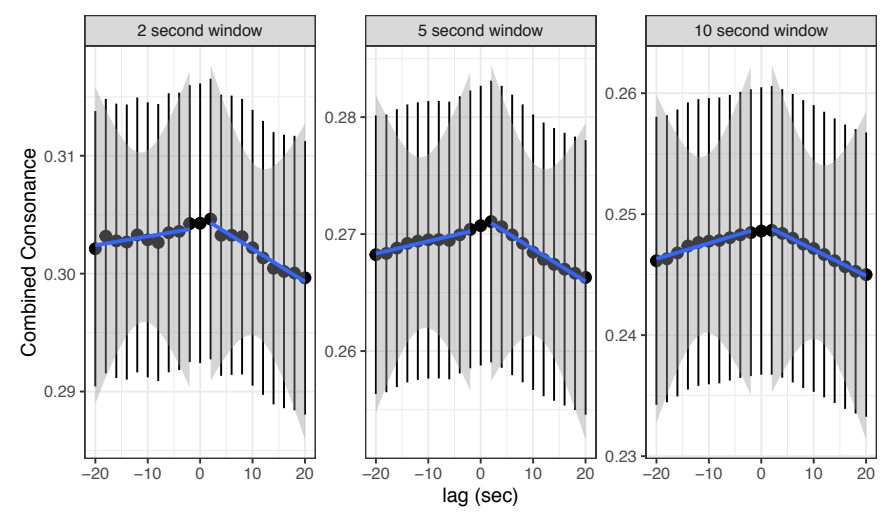

(d) Lagged combined consonance in coupled trials.

Fig. S10. Lagged consonance analysis. Live musicians harmonize with past notes of ghost recording significantly more so than the other way around. This effect is robust across a range of consonance window sizes and was found for both Emergent (a) and Combined (c) consonance measures. 


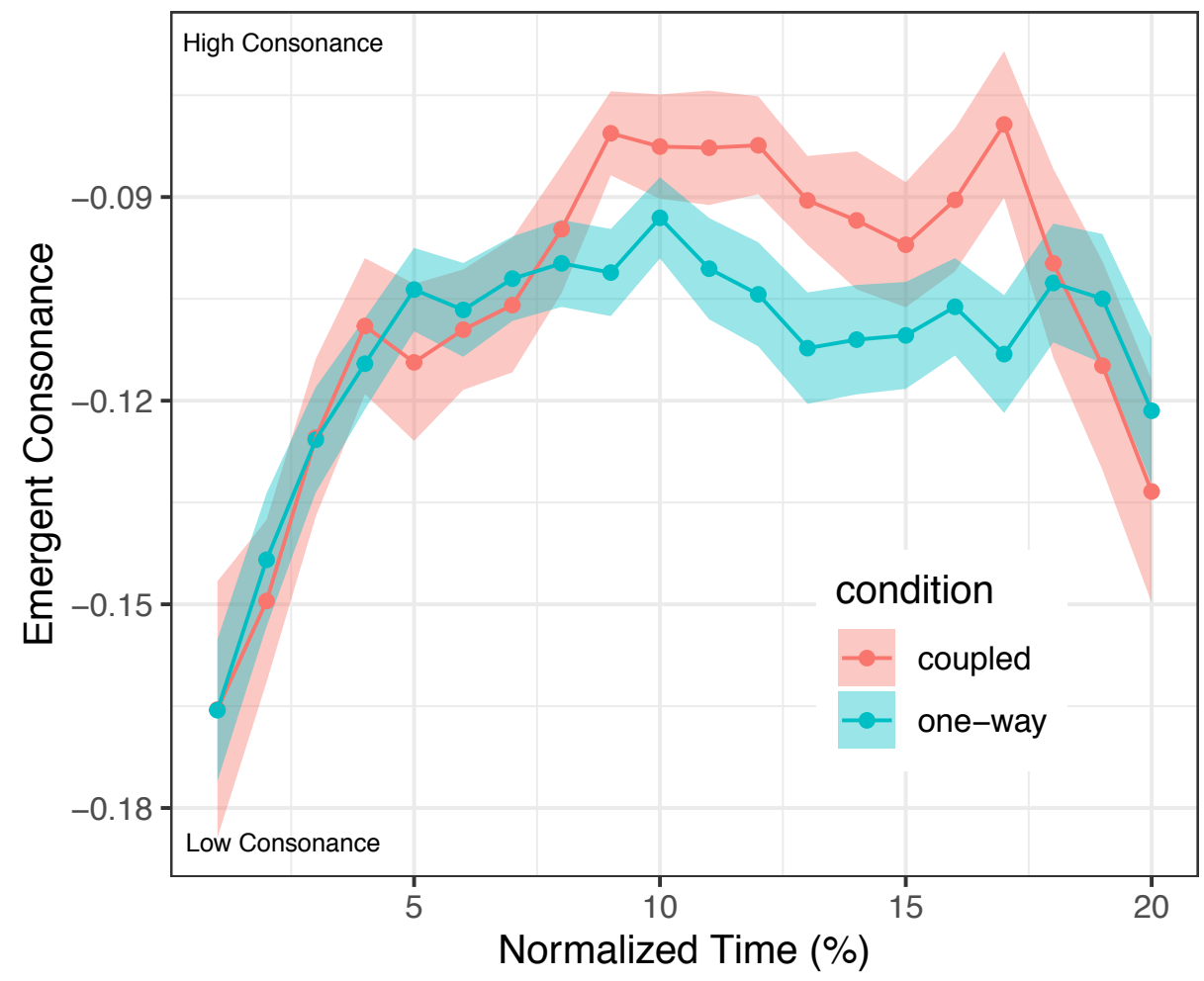

(a) 20 time bins

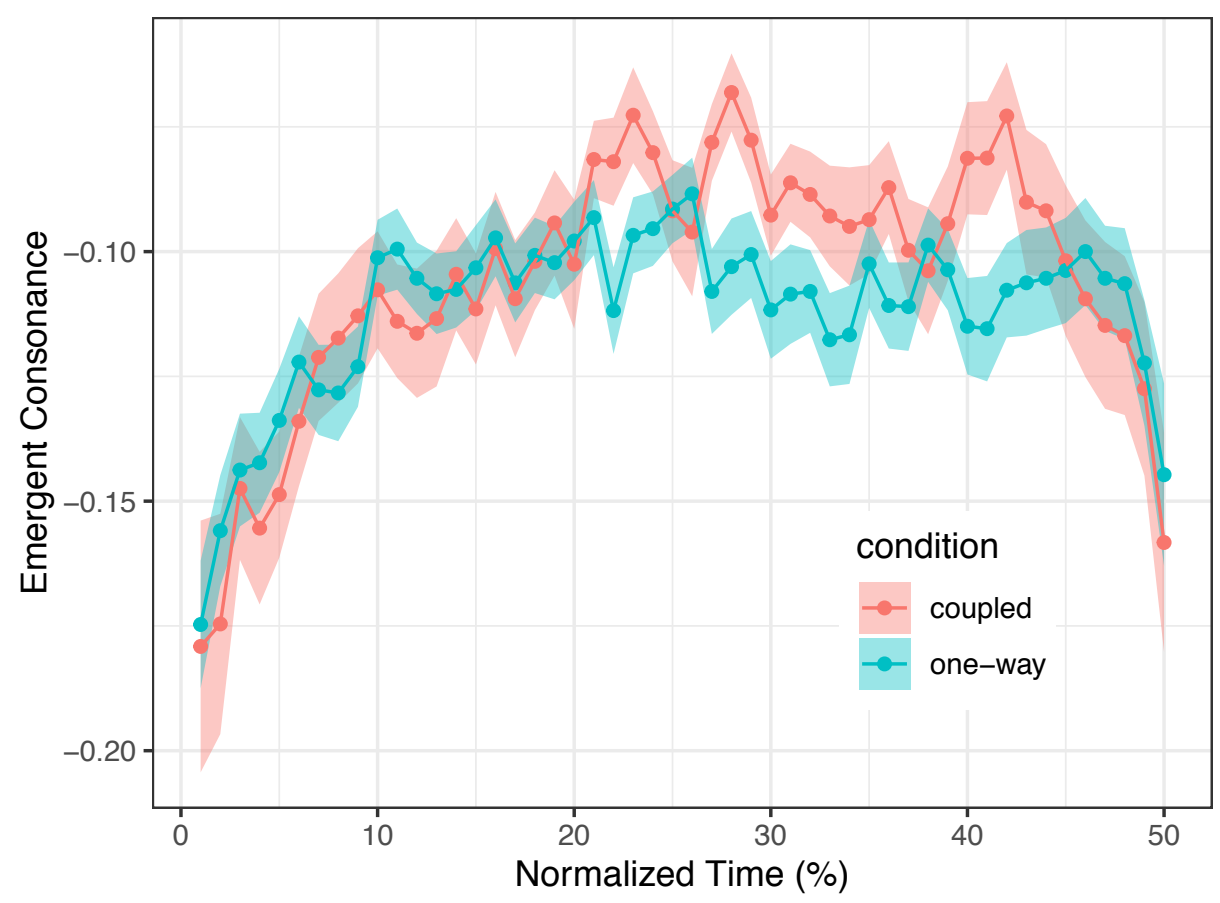

(b) 50 time bins.

Fig. S11. Emergent consonance over normalized time (5 second sliding window). 

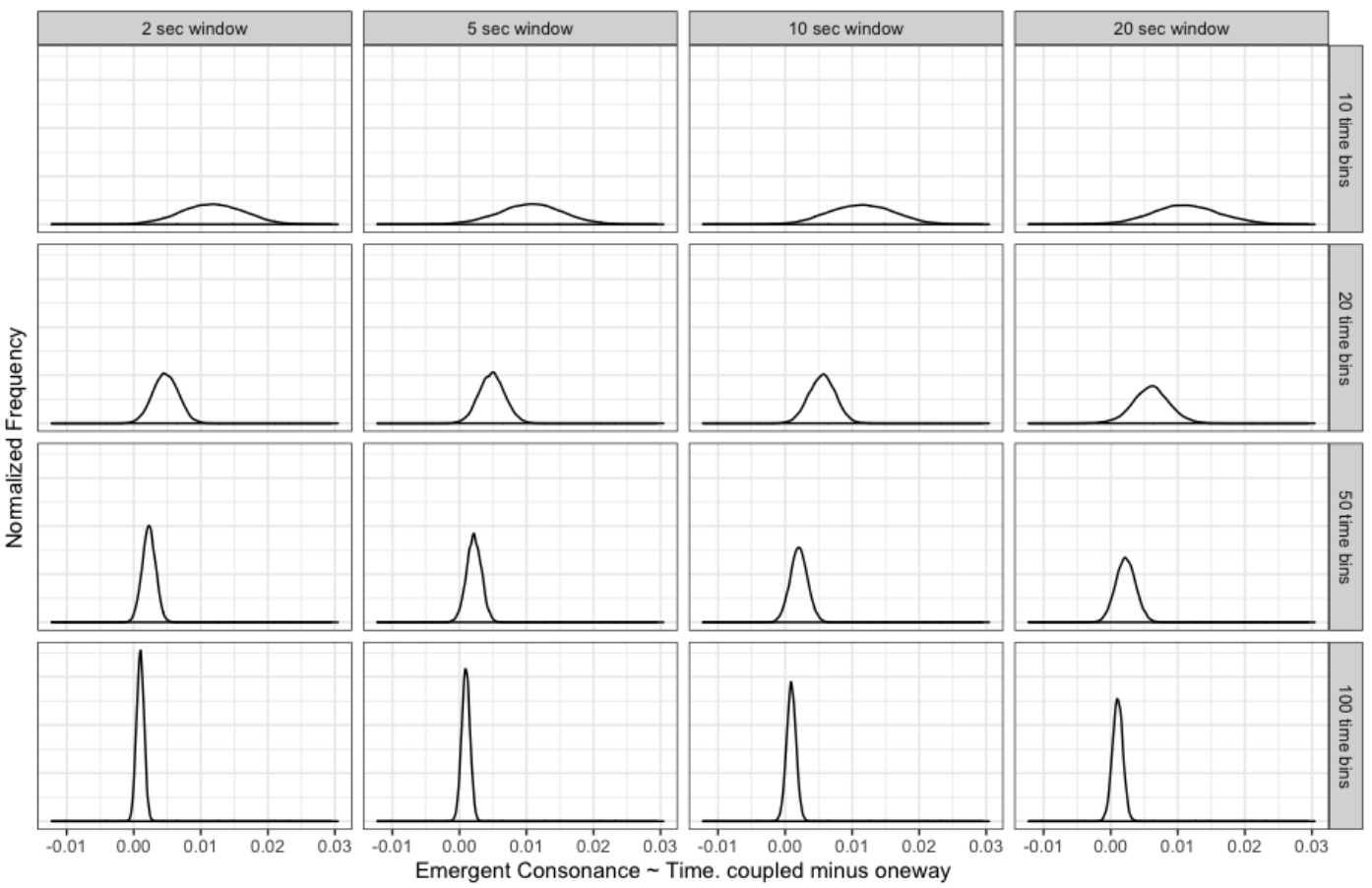

(a) Emergent consonance over time linear term.
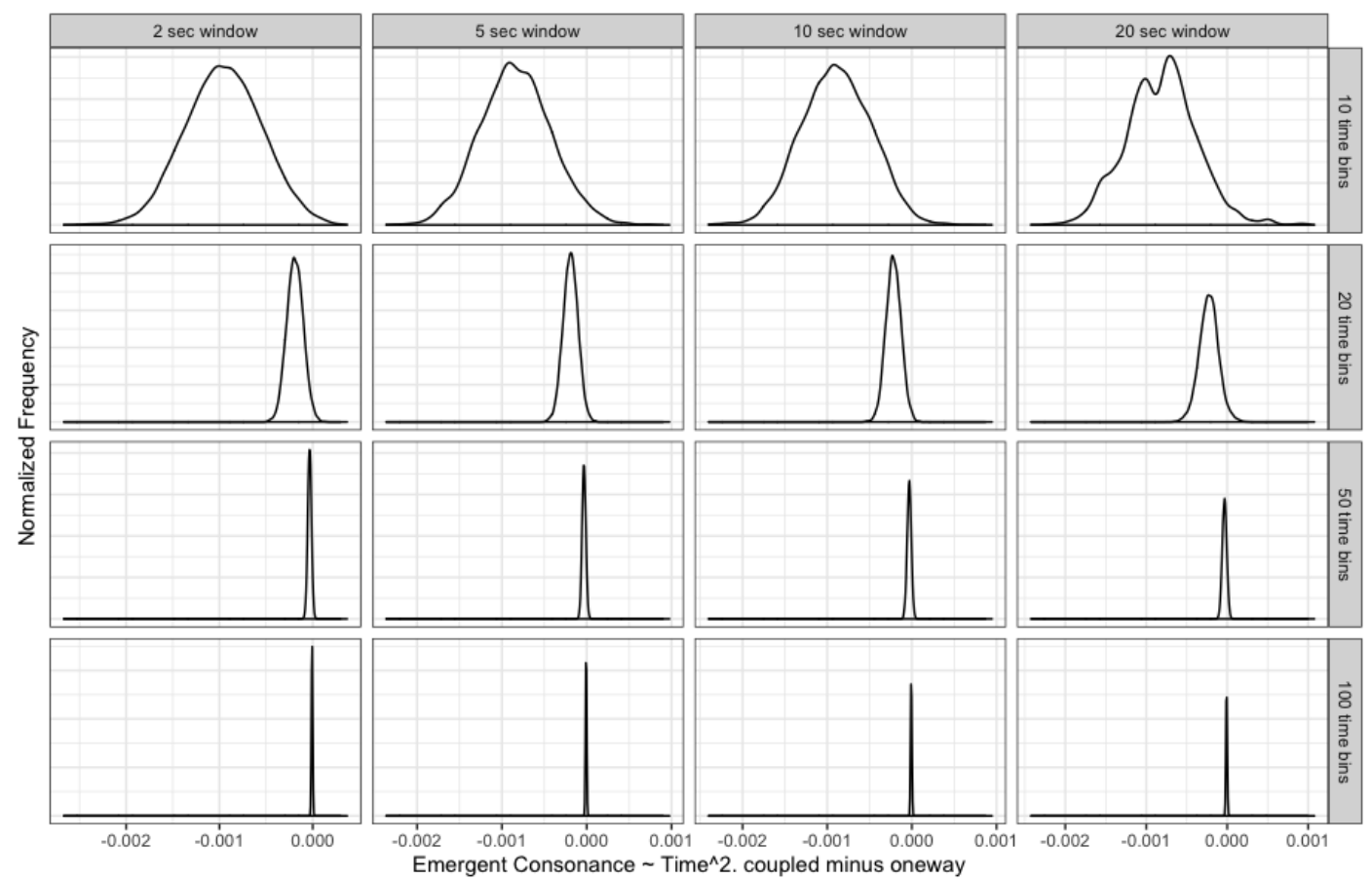

(b) Emergent consonance over time quadratic term.

Fig. S12. Posterior estimates for parameters of hierarchical model of Emergent Consonance over time. These are distributions of the difference in posterior estimates of means for condition-level sampling distributions (coupled minus one-way) for (a) linear (b) and quadratic terms. As shown in (a), the linear term is more positive for coupled trials indicating a greater tendency for tonal coordination to increase over improvised performance. This effect is robust across a range of window sizes and time binnings. 


\section{References}

1. E Chew, , et al., Mathematical and computational modeling of tonality. AMC 10, 12 (2014).

2. D Herremans, E Chew, , et al., Tension ribbons: Quantifying and visualising tonal tension. (2016).

3. P Harrison, M Pearce, Instantaneous consonance in the perception and composition of western music. (2019).

4. PN Johnson-Laird, OE Kang, YC Leong, On musical dissonance. Music. Perception: An Interdiscip. J. 30, 19-35 (2012).

5. E Chew, Regards on two regards by messiaen: post-tonal music segmentation using pitch context distances in the spiral array. J. New Music. Res. 34, 341-354 (2005).

6. L Barnett, AK Seth, The mvgc multivariate granger causality toolbox: a new approach to granger-causal inference. $J$. neuroscience methods 223, 50-68 (2014).

7. A Chang, SR Livingstone, DJ Bosnyak, LJ Trainor, Body sway reflects leadership in joint music performance. Proc. Natl. Acad. Sci. 114, E4134-E4141 (2017).

8. JJ Aucouturier, C Canonne, Musical friends and foes: The social cognition of affiliation and control in improvised interactions. Cognition 161, 94-108 (2017).

9. M Plummer, rjags: Bayesian graphical models using mcmc. $R$ package version 4 (2016).

10. JK Kruschke, Bayesian data analysis. Wiley Interdiscip. Rev. Cogn. Sci. 1, 658-676 (2010). 